\title{
Multiband Fitting to Three Long GRBs with Fermi/LAT Data: Structured Ejecta Sweeping up a Density-Jump Medium
}

\author{
S. Y. Feng ${ }^{1,2}$, Z. G. Dai ${ }^{1,2}$ \\ ${ }^{1}$ School of Astronomy and Space Sciences, Nanjing University, Nanjing 210093, China; \\ ${ }^{2}$ Key Laboratory of Modern Astronomy and Astrophysics (Nanjing University), Ministry of \\ Education, Nanjing 210093, China; \\ siyifeng@msn.com.dzg@nju.edu.cn
}

\begin{abstract}
We present broadband (radio, optical, X-ray and $\mathrm{GeV}$ ) fits to the afterglow light curves and spectra of three long-duration gamma-ray bursts (GRBs 080916C, 090902B, and 090926A) detected by the Gamma-Ray Burst Monitor (GBM) and Large Area Telescope (LAT) instruments on the Fermi satellite. Using the observed broadband data, we study the origin of the high energy emission, and suggest that the early-time $\mathrm{GeV}$ emission and the late-time radio, optical, and X-ray afterglows can be understood as being due to synchrotron emission from an external forward shock caused by structured ejecta propagating in a wind bubble jumping to a homogeneous density medium. If the ceasing time for majority of the energy injection is assumed to be close to the deceleration time of the forward shock, the structured ejecta with continuous energy injection to the forward shock can well explain the early rising feature of the $\mathrm{GeV}$ mission from these burst, and the density-jump medium can account for some certain plateaus or flares in the late afterglows. From our fits, we find that, on one hand, the external shock origin of the $\mathrm{GeV}$ photons will make the optical depth have not significant contribution to the early LAT rising part, which will loosen strong constraint of lower limits of Lorentz factor. On the other hand, these Fermi-LAT events preferentially occur in a low-density circumburst environment, in which case the Klein-Nishina cutoff will significantly suppress the Self-Synchrotron Compton (SSC) radiation. Such an environment might result from superbubbles or low-metallicity progenitor stars (which have a low mass-loss rate at late times of stellar evolution) of type $\mathrm{Ib} / \mathrm{c}$ supernovae.
\end{abstract}

Subject headings: gamma-rays: bursts — gamma-rays: theory 


\section{Introduction}

Gamma-ray bursts (GRBs) are a kind of astrophysical phenomenon so luminous in the universe that their isotropic energies of $10^{48}-10^{55} \mathrm{ergs}$ are usually considered coming from extremely relativistic outflows with bulk Lorentz factors as high as $10^{2}-10^{3}$. The connection between long-duration GRBs and broad-line SNe Ib/c (low-metallicity) has been supported by some pieces of observational evidence (Woosley \& Bloom 2006). The recently launched Fermi Gamma-Ray Space Telescope with the on-board Gamma-ray Burst Monitor (GBM) and Large Area Telescope (LAT) instruments (Atwood et al. 2009; Meegan et al. 2009) in conjunction with the Swift narrow field instruments (Gehrels et al. 2004) have opened a new era of understanding physical mechanisms of $\mathrm{GeV}$ photon emission in very energetic GRBs and their relation to lower-energy afterglow emission. Up to now, several novel yet tricky features have appeared during the whole period of observations and their complexities have challenged the anterior established theoretic models.

The first feature is that the sub-MeV and GeV photons observed by GBM and LAT respectively behave distinctive spectral and temporal properties. The GBM light curves are nearly flat during the main episode of the prompt emission, then drop extremely rapidly [e.g.

$t^{-3.3}$ for GRB 080916C (Abdo et al. 2009a)], and eventually cease abruptly. This can also be validated from $\sim 60 \%$ of all bursts detected by the Swift satellite (Evans et al. 2009). For the LAT light-curves, however, a rise appearing in the early few seconds $\left(<T_{90}\right)$ was followed by a single power-law decay without any cutoff up to hundreds of seconds after the GBM trigger, till below the monitor sensitivity, and decay of the late LAT emission was much shallower than the GBM-detected counterparts at the same times (Zhang et al. 2010). Furthermore, the LAT emission usually lags the GBM emission from a fraction of seconds to a few seconds. In addition, the spectral slopes of the GBM and LAT emissions are often different, e.g., the GBM data can be fitted with a Band spectrum that is composed of two smoothly-joining power laws, while the LAT data are often fitted by a power law with a slope intermediate between the two slopes of the GBM fit. These properties seem to indicate that the high-energy emission and low-energy emission detected by LAT and GBM have different origins.

The temporal properties of the LAT emission have been studied, and some explanations differing in the source's dominant component have been proposed. A prevalent explanation is particle-dominated models. One inclination is the leptonic interpretation. Because of their distinctive light curve properties we mentioned above, it is highly probable that the $\mathrm{MeV}$ photons may be of an internal origin, while the $\mathrm{GeV}$ photons may be generated via synchrotron emission of electrons accelerated by an external forward shock that also leads to lower-energy afterglow emission (Kumar \& Barniol Duran 2009). Wang et al. (2009, 2010) 
studied the Klein-Nishina effect on the high-energy afterglow emission and found that at early times such an effect strongly suppress the inverse Compton scattering of those electrons that produce the high-energy emission. Thus synchrotron radiation of the electrons becomes a plausible mechanism. This conclusion was independently drawn by Zhang \& Pe'er (2009), who suggested that the lack of a thermal component in the GBM spectrum of GRB 080916C is consistent with a relativistic Poynting-flux-dominated outflow. The explanation of synchrotron radiation of the electrons accelerated by a forward shock is fairly natural to account for both the observed delay of the $>100 \mathrm{MeV}$ photons and long lasting of their emission. However, a thermal component deviating from the smooth Band spectrum function in some bursts (i.e. GRB090510, GRB090902B, GRB090926A) seems to be beyond a prediction of what is often invoked from the external shock model (Zhang et al. 2010). Moreover, a rapid rise during the first few seconds (e.g. $\sim t^{6}$ of GRB080916C) is hard to be well explained within such a framework (Toma et al. 2009). Therefore, in addition to the hypothesis of a separate origin, Toma et al. (2009) assumed that $\mathrm{GeV}$ and $\mathrm{MeV}$ photons may come from the same region, but the onset of the high-energy may result from anisotropic inverse Compton scattering of an optical-thin expanding cocoon, delayed compared with the MeV emission. Nevertheless, in their calculation, this up-scattering cocoon is so short-lived that it could not account for the whole high energy emission.

Another approach is the hadronic scenario. Razzaque et al. (2009) suggested that the $\mathrm{MeV}$ and $\mathrm{GeV}$ photons could be interpreted as the radiation of accelerated electrons and cosmic ray protons respectively, as well as the delay between them could come from different cooling time scales in a highly magnetized shock. In their framework, the model of GRB 080916C is plausible only when $\Gamma \leq 500$ and the jet opening angle $\sim 1^{\circ}$. Alternatively, Asano et al. (2009) considered another possibility of the photomeson cascade and proton synchrotron models, and provided their constraint in GRB090510. Notwithstanding, to reproduce the extra component around $\mathrm{GeV}$ with these models, the isotropic-equivalent proton injection luminosity is required to be larger than $10^{55} \mathrm{erg} \mathrm{s}^{-1}$. Such a large proton luminosity is a challenge for the hadronic models.

All the works mentioned above inspire us to consider a plausible structured outflow, in which the bulk Lorentz factor of the initial shells tends to be lower than that of the late shells. This energy accumulation, therefore, would lead to an early rapidly rising light curve and transient soft to hard spectrum.

The second feature of the three long GRBs with LAT data is that some humps or flares appear in the light curves of the $>10^{5}$ s low-energy afterglows (Cenko et al. 2010; Swenson et al. 2010). Neglecting this feature, Kumar \& Barniol Duran (2010) fitted the multi-band light curves of these GRBs by assuming that a relativistic external shock sweeps 
up an interstellar medium, and obtained reasonable physical parameters or parameter spaces accordingly. Although their fittings somehow favor the external shock model, humps (or sometimes flares) have indeed been observed on the late-time optical and X-ray afterglow light curves, which do not completely accord with a simple power-law but instead the decay after the humps is shallower than that before the humps. These observations call for a more meticulous consideration of the external shock model.

It is noted that a density-jump medium proposed by Dai \& Lu (2002) has provided us with a clue for some optical and X-ray humps and well fitted to several bursts (Dai \& Wu 2003; Tam et al. 2005; Jin et al. 2009). In this scenario, a relativistic jet first expands in a stellar wind, subsequently encounters a density jump, and finally expands in a homogeneous medium. This interaction can produce an observed light-curve bump.

In this paper, we show that the early-time $\mathrm{GeV}$ emissions together with the late-time radio, optical, and X-ray afterglows of GRBs 080916C, 090902B, and 090926A can be understood as being due to synchrotron emission from an external forward shock caused by structured ejecta propagating in a wind bubble jumping to a homogeneous density medium. In Sec 2 we include a set of observed broadband (LAT, XRT, UVOT) data on these three luminous long bursts. Then, our model is set up in Sec 3. In particular, The structured ejecta can well explain the universal early rising feature of the GeV emission from these bursts, and the density-jump medium can account for some certain plateaus and flares in the late afterglows. Additionally, for the sake of verifying whether or not the lower energy (X-ray, optical, radio) emission originates from the same source as the higher energy (>100 MeV) emission, we discuss the effect of synchrotron self-Compton (synchrotron self-absorption) on the high energy (radio) emission, which, in the constrained parameter space estimated analytically from XRT and UVOT light curves, is proved in Sec4 to have a small contribution to the flux density during the observed period. In this section, we find a reasonable set of parameters valid for most of the late afterglows. Our conclusions concerning a plausible central engine and ambient environment of bright, long GRBs are discussed in Sec 5 .

Throughout this work, we adopt the convenience $Q_{x}=Q / 10^{x}$ in units of $\operatorname{cgs}$.

\section{Observations}

Among the 19 observed Fermi-LAT GRBs during the first 2 years' operation, GRB080916C, GRB090902B and GRB090926A are 3 typical brightest long GRBs with abundant of spectra information (Granot 2010). 


\subsection{GRB080916C}

This burst, located at redshift $z=4.35 \pm 0.15$ (Greiner et al. 2009), is the first GRB detected by Fermi with high significance of photons at energies $>0.1 \mathrm{GeV}$. The isotropic energy emitted from prompt emission can be estimated as $E_{\gamma, \text { iso }}=8.8 \times 10^{54} \mathrm{erg}$ (Abdo et al. 2009a). At 00:12:45.613542 on 16 September 2008 (Abdo et al. 2009a), this GRB was triggered on by GBM with the duration $T_{90}=66 \mathrm{~s}$. Before $T_{0}+6 \mathrm{~s}$, the LAT light-curve shows an extremely steep rise $F_{\nu, L A T} \propto t^{6 \pm 0.5}$, followed by a simple power law decay $F_{\nu, L A T} \propto t^{-1.33 \pm 0.08} \nu^{-1.1 \pm 0.1}$ until $\sim$ 1400s (Zhang et al. 2010). X-ray and optical photons were detected by XRT and UVOT since 17:11:28 16 September $2008\left(T_{0}+61 \mathrm{ks}\right)$. A steep decay (to $\sim T_{0}+101 \mathrm{ks}$ ) continued with a plateau (to $\sim T_{0}+204 \mathrm{ks}$ ) goes to a slightly shallower decay without break until $1.3 \mathrm{Ms}$ from the trigger $T_{0}$. During the period of $T_{0}+61 \mathrm{ks}-T_{0}+1306 \mathrm{ks}$, the simple power law light curve and spectrum evolution show the flux density at X-ray band, $F_{\nu, X} \propto t^{-1.29 \pm 0.09} \nu^{-0.50 \pm 0.16}$, as well as for the optical band, $F_{\nu, o p t} \propto t^{-1.40 \pm 0.05} \nu^{-0.38 \pm 0.20}$ (Greiner et al. 2009). Besides, AGILE, RHESSO, INTEGRAL, Konus-Wind, and MESSENGER all provide a plentiful of data information for the late afterglow (Perri et al. 2008).

Many groups have studied this GRB with the external shock model (Kumar \& Barniol Duran 2010; Gao et al. 2009; Zou et al. 2009), and suggested that the late time afterglow data can be used to extrapolate the early LAT data as well as be predicted from it, under the preference of circumburst density stratification.

\subsection{GRB090902B}

This burst is an exceptional case with redshift $z=1.822$, whose speciality lied in both phases of prompt emission and early afterglow (Abdo et al. 2009b). Unlike majority of the other GRB events, excess emission exhibited in both low $(\leq 100 \mathrm{keV})$ and high $(\geq$ $10 \mathrm{MeV}$ ) band during the prompt emission. Moreover, the soft-hard-soft spectral evolution indicates two components: Band function peaking at $\sim 700 \mathrm{keV}+$ simple power-law with photon index $\Gamma \sim 1.85$ (Zhang et al. 2010). The isotropic energy release $E_{\gamma, \text { iso }}=(3.83 \pm$ $0.05) \times 10^{54} \mathrm{erg}$ (Cenko et al. 2010). There are some explanations for origin of the highenergy power-law emission component observed in the LAT energy range (which accounts for $\sim 24 \%$ of the total $10 \mathrm{keV}$ to $10 \mathrm{GeV}$ fluence), including the hadronic origin [either proton synchrotron radiation (Razzaque et al. 2009) or photohadronic interactions (Asano et al. 2009)], or thermal emission from the jet photosphere (Ryde et al. 2010).

This burst was triggered and located at 11:05:08.31 on September 2009, by the FermiGBM (Bissaldi \& Connaughton 2009) and Suzaku-WAM (Terada et al. 2009) with multi- 
peaked duration $T_{90} \approx 21 s$. After a rapid rise until $T_{0}+7 \mathrm{~s}$, the LAT band light-curve decays up to $T_{0}+1 \mathrm{ks}$ with $F_{\nu, L A T} \propto t^{-1.4 \pm 0.06}$, including an energetic photon detected as high as $11.16_{-0.58}^{+1.48} \mathrm{GeV}$ within the prompt emission phase and another $33.4_{-35}^{+3.7} \mathrm{GeV}$ at $T_{0}+82 \mathrm{~s}$ (Abdo et al. 2009b). The Swift XRT (Kennea \& Stratta 2009; Evans et al. 2009b; Stratta et al. 2009) and UVOT (Swenson \& Siegel 2009) began concurrently target of opportunity of the field of GRB090902B's fading source from 23:36 on 2 September 2009 and copious of data have been in hand from $T_{0}+0.5 \mathrm{ks}$. A steep decay (to $\sim T_{0}+116 \mathrm{ks}$ ) simultaneously ended with a slight rise in both bands. It is highly possible that jet break occurred at $\sim T_{0}+553 \mathrm{ks}$ (Cenko et al. 2010). During this whole period, the X-ray spectrum evolved as $F_{\nu, X} \propto t^{-1.36 \pm 0.03} \nu^{-0.90 \pm 0.13}$, and for the optical band, $F_{\nu, o p t} \propto t^{-0.89 \pm 0.05} \nu^{-0.76 \pm 0.07}$. In addition, VLA began to observe the afterglow since 3 September 2009 (Chandra \& Frail 2009) until 5 months later at $8.5 \mathrm{GHz}$ and $4.8 \mathrm{GHz}$.

Using the forward-reverse shock and constant density model, Cenko et al. (2010) suggested the afterglow of this burst is better fitted in X-ray and radio bands than in the optical/UV band, except for the first point at $\sim 10^{4} \mathrm{~s}$. Parameter constraints without taking LAT data into account suggest a low circumburst density and a large kinetic energy, which is possible in the narrow opening angle $\sim 3.4^{\circ}$. Later, Liu \& Wang (2010) have provided a meticulous discussion about the two-component forward-reverse external shock in a monotonic circumburst environment and their calculation was well fit to the four observed bands, except for the very early rising part of the LAT light curve.

\subsection{GRB090926A}

This burst is the GRB detected at $z=2.1062$ by Fermi with photons as high as $\sim 20$ $\mathrm{GeV}$ at $26 \mathrm{~s}$ after the trigger (Uehara et al. 2009). The prompt emission time-integrated flux indicates its isotropic emission energy of $E_{\gamma, \text { iso }}=2.1_{-0.08}^{+0.09} \times 10^{54} \mathrm{erg}$ (Zhang et al. 2010).

Since the trigger at 04:20:26:26.99 on 26 September 2009, GBM and LAT started operation and found that multiple pulses with total duration $T_{90} \approx 20 \mathrm{~s}$ can be well modeled by a Band function spectrum in the prompt emission, along with the Suzaku-WAM (Noda et al. 2009), Konus-Wind (Golenetskii et al. 2009), and RT-2 on CORONAS-PHOTON (Chakrabarti et al. 2009). The light curve rose slightly for quite a few seconds (to $\sim T_{0}+16 \mathrm{~s}$ ), and decayed $F_{\nu, L A T} \propto t^{-2.05 \pm 0.14} \nu^{-1.26_{-0.22}^{+0.24}}$. A fading X-ray counterpart was observed by XRT since 17:17 on 26 September $\left(T_{0}+46.7 \mathrm{ks}\right.$ ) (Vetere et al. 2009), then PROMPT, SMARTS detected photons in several optical filters but no radio $(5.5 \mathrm{GHz})$ source under the limit of $1.5 \mathrm{mJy}$ was detected up to 1 October 2009. As shown on the light curve, a plateau $\left(T_{0}+51.4 \mathrm{ks}\right.$ $\sim T_{0}+92 \mathrm{ks}$ ) overlaps the simple power law decay that is slightly steeper than the fading 
supposed to be extrapolated from the early trace. No break up to 100ks from the trigger $T_{0}$ was observed. During the period from $T_{0}+46.7 \mathrm{ks}$ to $T_{0}+149 \mathrm{ks}$, two variabilities, are suggested to be flares (the first, at $\sim 70 \mathrm{ks}-95 \mathrm{ks}$ with $\delta t / t \approx 0.35$; the second is slight, at $195 \mathrm{ks}-260 \mathrm{ks}$, with $\delta t / t \approx 0.28$ ) overly on the simple power law light curve in both X-ray band $\left(F_{\nu, X} \propto t^{-1.40 \pm 0.05} \nu^{-1.6_{-0.2}^{+0.3}}\right)$ and optical band $\left(F_{\nu, o p t} \propto t^{-1.01_{-0.07}^{+0.03}}\right)$ (Swenson et al. 2010$)$.

Cenko et al. (2010) and Rau et al. (2010) studied the later afterglow (X-ray, optical) of this burst, provided a certain parameter space, confirmed the second flare, and indicated that the jet break occurs around $\sim 21 \mathrm{~d}$. However, due to lack of radio data, the constraints cannot be narrowed down. Meanwhile, as for a common rebrightening plateau in 5 optical bands, they suggested a density-jump circumstance (e.g., Dai \& Lu 2002; Lazzati et al. 2002; Tam et al. 2005) or a smooth injection of energy into the forward shock from the central engine (e.g. Dai \& Lu 1998a; Rees \& Meszaros 1998), and called for a detailed analysis for the early higher energy emission.

\section{Model}

The behavior of the GRB afterglows shed light on the external shock model (Panaitescu \& Kumar 2002). A collimated $\left(\theta \leq 10^{\circ}\right)$, ultrarelativistic outflow of matter and/or radiation is driven by a central engine. Some certain dissipative processes within the outflow give rise to the prompt gamma-ray emission, with a fraction $\varsigma_{\gamma} \equiv E_{\gamma, \text { iso }} /\left(E_{\gamma, \text { iso }}+E_{K, \text { iso }}\right)$ of the total relativistic energy converted to high-energy radiation.

A self-consistent result of the multiband (X-ray, optical, radio) afterglow data coordinated with the prompt emission relics producing $>100 \mathrm{MeV}$ photons, may support the external shock origin of LAT photons, which is the same as the later lower-band emission. Meanwhile, due to the difference between the light curve slope of LAT and GBM, we take no consideration of the GBM emission in this piece. Moreover, the particularities of GRB 090902B we mentioned above (soft-hard-soft spectral evolution, flares) may evoke doubts about the conventional external shock + monotonic circumstance model (we will discuss it in Sec 5), but enlighten our deliberate consideration on amendment to structured ejecta sweeping up the density-jump medium. 


\subsection{Dynamics}

\subsubsection{Before the Deceleration Time}

Energy can be injected by a central engine continuously during a period (majority of which is within the first main pulse of prompt emission, before $t_{f} \frac{1}{1}$ ). We here suggest, within the framework of the collapsar model, an increase of the Lorentz factor of the ejecta in active time of the central engine may due to two reasons. First, angular momentum of the accreted fall-back matter would spin up the central compact object and thus its rotational energy loss could give rise to an increase of the ejecting luminosity. Second, earlier-ejected shells, when breaking through the stellar envelope, may suffer from more massive baryon contamination, and thus later-ejected shells may propagate in a tunnel (the later the cleaner). Therefore, the Lorentz factor of the ejecta head may increase with time. For simplicity, we assume the bulk Lorentz factor of the front materials distribute as a power-law function of time: $\Gamma \propto t^{\varkappa}$ for $t \leq t_{f p}$. If $\varkappa>0$, the Lorentz factor of the shocked matter increases, being due to energy injection.

We denote $M_{\text {ej }}$ as the accumulated mass ejected by the central engine at some certain time, and $\eta$ as the bulk Lorentz factor of a blast wave at this certain time, which is defined when the mass of the surrounding matter swept up by the blast wave, $M_{\mathrm{sw}}$, is equal to $M_{\mathrm{ej}} / \eta$. After this time, the bulk Lorentz factor presents an obvious deceleration, contributing to a peak of the LAT light curve. This time is called the deceleration time $t_{d e c}$, which is assumed in this paper to be around the stop time of effective energy injection. This assumption is reasonable because energy injection to the forward shock is effected by the circumburst medium. Thus, both the apparent energy-injection time $t_{f p}$ and the deceleration time $t_{d e c}$ should be shorter than the central engine ceasing time $T_{90}$.

The shock propagates a distance $\delta R \sim 2(1+z)^{-1} \Gamma^{2} c \delta t$ during a small observed time

$\delta t$. Assuming the proton number density of a medium $n \propto R^{-k}$, thus for a homogeneous medium $(k=0), n=$ const (Sari et al. 1998) or for a wind medium $(k=2), n=A R^{-2}=$

\footnotetext{
${ }^{1}$ As Fig. 2, 10, 11 in Zhang et al. (2010) shown, majority of the $>100 \mathrm{MeV}$ photons in the prompt emission are released during the first largest pulse (at $\sim 10 \mathrm{~s}, \sim 10 \mathrm{~s}, \sim 10 \mathrm{~s}$ for GRB 080916C, GRB 090902B, and GRB 090926A, separately). Comparing this time to the peaking time of the LAT lightcurve $(\sim 6 \mathrm{~s}, \sim$ $7 \mathrm{~s}, \sim 16 \mathrm{~s}$ for GRB 080916C, GRB 090902B, and GRB 090926A, separately), and the $T_{90}(\sim 66 \mathrm{~s}, \sim 21 \mathrm{~s}, \sim 20 \mathrm{~s}$ for GRB 080916C, GRB 090902B, and GRB 090926A, separately), we defined $t_{f p}$, before when energy has been largely injected. Of course, as mentioned in Maxham et al. (2010), whole of the energy injection last longer, while it will dwindle after the first largest pulse.
} 
$10^{35.5} A_{35.5} R^{-2}$ (Dai \& Lu 1998b; Chevalier \& Li 2000). In both cases, we obtain

$$
t_{\mathrm{dec}}=\frac{(2 \varkappa+1)(1+z)}{2 \eta^{2} c} R_{\mathrm{dec}}
$$

The deceleration radius can be written as

$$
R_{\mathrm{dec}}=\left(\frac{3-k}{4 \pi A m_{p}} \frac{E_{\mathrm{K}, \mathrm{iso}}}{\eta^{2} c^{2}}\right)^{1 /(3-k)} .
$$

Therefore, $R\left(t \leq t_{d e c}\right)=R_{\text {dec }}\left(t / t_{\mathrm{dec}}\right)^{2 \varkappa+1}$. According to Sari (1997), in order to check whether the the unshocked ejecta is assumed to be equal to that of the shocked matter, let $\triangle=c t_{f p}(1+z)^{-1}$ represent the thickness of the shell, and $f$ be the density ratio between the preshock fluid in the shell and in the circumburst surrounding $(k=2)$, given by

$$
f=\frac{(1+\varkappa)(1+z) E_{\mathrm{K}, \text { iso }}}{4 \pi A R^{2-k} m_{p} c^{3} \eta^{2}} \frac{t_{f p}{ }^{\varkappa+1}}{t^{\varkappa+2}}, \quad t \leq t_{f p}
$$

This may bring about a correction for the $\mathrm{GeV}$ rising part under different conditions, i.e., in the thick shell case, $f \ll \Gamma^{2}$, a reverse shock is relativistic, the Lorentz factor of the shocked fluid should be changed and the contribution of the reverse shock to the flux density should be considered, while in the thin shell case, $f \succsim \Gamma^{2}$, this change is negligible. However, no matter how the thickness of the shell is, $t_{f p} \leq t_{d e c}$. In the following analytical calculations, we simplify that $t_{f p} \sim t_{\text {dec }}$ (which can be observed from the figures of temporal flux density) and assume that the self-similar condition is established after the deceleration time.

Before the deceleration time, energy is continuously injecting into the former shells. The mass ejected later catches up with the earlier ones, and $\Gamma$ can be simplified as the bulk Lorentz factor of these materials combination. In the very beginning, it is possible that the bulk Lorentz factor is too low for majority of high-energy gamma rays to escape from the MeV background. According to predominate hypothesis (Granot 2010), only when the optical depth of the $\gamma$-ray absorption $\tau_{\gamma \gamma}<1$, the bulk Lorentz factor is large enough to radiate almost completely. In this piece, we use sketchy in Zou et al. (2011) to check whether the absorption play a dominate role in certain parameter spaces.

\subsubsection{In the Density-Jump Surrounding}

Because of the association of long GRBs with star forming regions, it is highly possible that massive stars are still embedded in a cloud when giving birth to GRBs. Low density bubbles are created by stellar winds from GRB progenitors, whose sizes and densities strongly 
depend on the initial ambient density. Therefore, a density jump occurs at the boundary between the wind bubble and the outer cloud, which may result in a bump/flare and shallow decay of the light curve during the later period (Tam et al. 2005).

From the moment $t_{\text {break }}$ at which the blast wave reaches the boundary, to the termination when it comes out into the interstellar medium at $t_{\text {end }}$, the number density of the medium changes as

$$
n=A R^{-k}= \begin{cases}3 \times 10^{35} A_{35.5} R^{-2}, & R \leq R_{\text {break }}, \\ 3 \times 10^{35}\left[1+\frac{\log R-\log R_{\text {break }}}{\log R_{\text {end }}-\log R_{\text {break }}}(\xi-1)\right] A_{35.5} R_{\text {break }}^{-2}, & R_{\text {break }} \leq R \leq R_{\text {end }}, \\ n_{\text {after }} \equiv 3 \times 10^{35} \xi A_{35.5} R_{\text {break }}^{-2}, & R \geq R_{\text {end }},\end{cases}
$$

where $\xi$ is the ratio of the number density in front of and behind the jump.

It has been usually assumed that the afterglow emission from the blast wave is nearly adiabatic (Mészáros \& Rees 1997; Sari et al. 1998), so the total kinetic energy of the relativistic shock is constant. However, if we take radiative energy loss into consideration, the radiation efficiency of the blast wave can be given with the electronic fraction $\epsilon_{e}$ by $\mathrm{Wu}$ et al. $(2005)$

$$
\varepsilon=\left\{\begin{array}{lr}
\epsilon_{e}, & \nu_{c} \leq \nu_{m}, \\
\left(\frac{\nu_{m}}{\nu_{c}}\right)^{(p-2) / 2}, & \nu_{m} \leq \nu_{c} .
\end{array}\right.
$$

In such a situation, energy loss is significant in the fast cooling and gradually dwindle to the quasi-adiabatic case (i.e., the slow cooling phase).

Hereafter, a denotation $m=(3-k) /(1-\varepsilon)$ can analytically result in the bulk Lorentz factor evolution,

$$
\Gamma= \begin{cases}\eta\left(\frac{t}{t_{\mathrm{dec}}}\right)^{\varkappa}, & t \leq t_{\mathrm{dec}} \\ \eta\left(\frac{t}{t_{\mathrm{dec}}}\right)^{-m /(2 m+2)}, & t \geq t_{\mathrm{dec}}\end{cases}
$$

Consequently, the Doppler factor becomes $\mathcal{D}=\frac{1}{\Gamma(1-\beta \cos \theta)}=\frac{2 \Gamma}{1+\Gamma^{2} \theta^{2}} \sim 2 \Gamma$ (where $\theta$ is the emitting latitude angle).

When the blast wave reaches the density boundary, the observed light curve will not behave as an abrupt jump but a smooth rise or plateau instead, due to the curvature effect (Fenimore et al. 1996; Kumar \& Panaitescu 2000). Although this is trifle to be accomplished in analytic modeling, a simplified power law distribution of medium density in the period of $t_{\text {break }} \sim t_{\text {end }}$ can account for the same light curve feature (See Fig. 4,a). Succeedingly, the blast wave radius can be written as

$$
R= \begin{cases}R_{\mathrm{dec}}\left(\frac{t}{t_{\mathrm{dec}}}\right)^{2 \varkappa+1}, & t \leq t_{\mathrm{dec}} \\ R_{\mathrm{dec}}\left(\frac{t}{t_{\mathrm{dec}}}\right)^{(1-\varepsilon) /(4-k-\varepsilon)}, & t \geq t_{\mathrm{dec}}\end{cases}
$$




\subsection{Radiation}

In the comoving frame, with an electronic fraction $\epsilon_{e}$ and magnetic fraction $\epsilon_{B}$ of the post-shock thermal energy density, which may either vary or not with different media. Denote $f_{p} \equiv 6(p-2) /(p-1)$, the magnetic field intensity $B=\Gamma\left(32 \pi \epsilon_{B} n m_{p} c^{2}\right)^{1 / 2}$. The cooling, minimum, and maximum electron Lorentz factors are $\gamma_{c}=\gamma_{s y n, c} /\left[1+Y\left(\gamma_{c}\right)\right]=$ $6 \pi m_{e} c(1+z)\left(\sigma_{T} B^{2} \Gamma t\right)^{-1} /\left[1+Y\left(\gamma_{c}\right)\right], \gamma_{m}=(1 / 6) f_{p} \epsilon_{e}\left(m_{p} / m_{e}\right) \Gamma$, and $\gamma_{\max } \sim 10^{8} / \sqrt{B}$, while the synchrotron characteristic frequencies $\nu_{c}, \nu_{m}, \nu_{\max }$ can be calculated by $\nu_{i}=$ $\gamma_{i}^{2} \mathcal{D} e B /\left[2 \pi m_{e} c(1+z)\right]$ (where $i=c, m, \max$ ), where $Y\left(\gamma_{*}\right)$ is the Compton factor for electrons with the Lorentz factor $\gamma_{*}$, defined as the ratio of the self-inverse Compton (SSC) to synchrotron emission (Nakar et al. 2009).

$$
Y\left(\gamma_{*}\right) \equiv \frac{P_{S S C}\left(\gamma_{*}\right)}{P_{s y n}\left(\gamma_{*}\right)}
$$

When Klein-Nishina (KN) effects are unimportant, i.e., the IC scattering of $\gamma_{*}$ electrons with synchrotron photons are in the Thomson scattering regime, $Y\left(\gamma_{*}\right)$ can be simply derived by (Sari \& Esin 2001) as $\left(\sqrt{1+4 \varepsilon \epsilon_{e} / \epsilon_{B}}-1\right) / 2$, which is independent with $\gamma_{*}$. However, for high-energy electrons whose KN effect becomes important, $Y\left(\gamma_{*}\right)$ depends on $\gamma_{*}$ and can be expressed as analytical solutions in different cases from Wang et al. (2010).

The total number of shocked accelerated electrons $N_{e}=4 \pi n R^{3} /(3-k)$. The peak spectral power of a single electron is $P_{\nu, \max }=\sigma_{T} m_{e} c^{2} \mathcal{D} B /(3 e)$, and the peak flux density at certain time of the afterglow is $F_{\nu, \max }=(1+z) N_{e} P_{\nu, \max } /\left(4 \pi D_{L}^{2}\right)$. Here, the luminosity distance $D_{L}=(1+z) H_{0}{ }^{-1} c \int_{0}^{z}\left[\Omega_{m}(1+z)^{3}+\Omega_{k}(1+z)^{2}+\Omega_{\Lambda}\right]^{-1 / 2} d z$ (here we adopt a standard $\Lambda$ CDM cosmology with $H_{0}=71 \mathrm{kms}^{-1} \mathrm{Mpc}^{-1}, \Omega_{m}=0.27, \Omega_{k}=0.0, \Omega_{\Lambda}=0.73$ (Spergel et al. 2007)).

For a lower energy band (e.g., radio), synchrotron self-absorption may play a crucial role. The self-absorption optical depth $\tau_{\nu, m}$ or $\left(\tau_{\nu, c}\right)$, according to the definition $\tau_{\nu}=1$, becomes $\tau_{\nu, m}=\frac{c_{0}(p-1)}{(3-k)} \frac{e n R}{B \gamma_{m}{ }^{5}}$ and $\tau_{\nu, c}=\frac{c_{0}}{(3-k)} \frac{e n R}{B \gamma_{c}{ }^{5}}$, where $c_{0} \approx 10.4(p+2) /(p+2 / 3)(\mathrm{Wu}$ et al. 2005). Succeedingly, the self-absorption frequency $\nu_{a}$ can be written as

$$
\nu_{a}= \begin{cases}\tau_{\nu, l}^{3 / 5} \nu_{l}, & \tau_{\nu, l}<1, \\ \tau_{\nu, l}^{2 /(p+4)} \nu_{l}, & 1 \leq \tau_{\nu, l}<\left(\frac{\nu_{h}}{\nu_{l}}\right)^{(p+4) / 2} \\ \tau_{\nu, l}^{2 /(p+5)}\left(\frac{\nu_{h}}{\nu_{l}}\right)^{1 /(p+5)} \nu_{l}, & \left(\frac{\nu_{h}}{\nu_{l}}\right)^{(p+4) / 2} \leq \tau_{\nu, l}<\left(\frac{\nu_{\max }}{\nu_{l}}\right)^{(p+5) / 2}\left(\frac{\nu_{l}}{\nu_{h}}\right)^{1 / 2}\end{cases}
$$


Then, the SSA power-law light curve yields (Sari \& Esin 2001)

$$
F_{\nu}=F_{\nu, \max } \begin{cases}\left(\frac{\nu_{a}}{\nu_{l}}\right)^{1 / 3}\left(\frac{\nu}{\nu_{a}}\right)^{2}, & \nu \leq \nu_{a} \\ \left(\frac{\nu}{\nu_{l}}\right)^{1 / 3}, & \nu \leq \nu_{l} \\ \left(\frac{\nu}{\nu_{l}}\right)^{-(q-1) / 2}, & \nu_{l} \leq \nu \leq \nu_{h} \\ \left(\frac{\nu_{h}}{\nu_{l}}\right)^{-(q-1) / 2}\left(\frac{\nu}{\nu_{h}}\right)^{-p / 2}, & \nu \geq \nu_{h}\end{cases}
$$

Here $\nu_{l}=\min \left(\nu_{m}, \nu_{c}\right), \nu_{h}=\max \left(\nu_{m}, \nu_{c}\right) . q=2$ when $\nu_{c}<\nu_{m}$, while $\nu_{c}>\nu_{m}, q=p$.

It is noted that before the optical depth becomes thin, the annihilation effect of highenergy photons can affect their flux, so the observed flux can be estimated as $F_{\mathrm{ob}, \nu}=e^{-\tau_{\gamma \gamma}} F_{\nu}$. After $T_{90}, F_{\mathrm{ob}, \nu}=F_{\nu}$.

For a high energy band, synchrotron self-Compton (SSC) radiation might contribute to the observed flux, the characteristic frequencies $\nu_{I C, c}, \nu_{I C, m}, \nu_{I C, \max }, F_{I C, \nu, \max }$ can be calculated by $\nu_{I C, i}=2 \nu_{i} \gamma_{i}^{2}$ (where $i=c, m, \max$ ), and $F_{I C, \nu, \max }=(14 / 45) \sigma_{T} R n F_{\nu, \max }$. Thus the SSC power-law light curve yields (Gupta \& Zhang 2007)

$$
F_{\nu, I C}=F_{I C, \nu, \max } \begin{cases}\left(\frac{\nu}{\nu_{I C, l}}\right)^{1 / 3}, & \nu \leq \nu_{I C, l}, \\ \left(\frac{\nu}{\nu_{I C, l}}\right)^{-(q-1) / 2}, & \nu_{I C, l} \leq \nu \leq \nu_{I C, h} \\ \left(\frac{\nu_{I C, h}}{\nu_{I C, l}}\right)^{-(q-1) / 2}\left(\frac{\nu}{\nu_{I C, h}}\right)^{-p / 2}, & \nu_{I C, h} \leq \nu\end{cases}
$$

Here $\nu_{I C, l}=\min \left(\nu_{I C, m}, \nu_{I C, c}\right), \nu_{I C, h}=\max \left(\nu_{I C, m}, \nu_{I C, c}\right) . q=2$ when $\nu_{I C, c}<\nu_{I C, m}$, while $\nu_{I C, c}>\nu_{I C, m}, q=p$.

However, due to the KN effects on optically thin spectrums, Equations (10) and (11) should be amended as different cases mentioned in equations of Nakar et al. (2009). This calls for the definition of electron KN Lorentz factor: $\widehat{\gamma}_{i}=m_{e} c^{2} \Gamma /\left[h \nu_{s y n}\left(\gamma_{i}\right)\right](i=c, m)$. We have, if the synchrotron photons emitted by electrons with Lorentz factor larger than $\gamma_{i}$ [i.e. with frequency $>\nu_{\text {syn }}\left(\gamma_{i}\right)$ ], they cannot be upscattered efficiently by electrons with Lorentz factor larger than $\widehat{\gamma}_{i}$ (which is above the KN limit).

In the case of a jet, a simple assumption is that the jet decelerates without sideways expansion. After the jet break time $t_{j e t}$ at which the jet angle $\theta_{j} \sim 1 / \Gamma$ (where the jet axis is assumed to be along the line of sight), the observed flux $F_{\nu \text {,jet }}=F_{\nu}\left(t / t_{j e t}\right)^{(3-k) /(4-k)}$.

Comparing the flux density estimated analytically above with the observational multiband values, certain physical parameters can be constrained. Whether the parameter space is reasonable or not can on some extent verify the feasibility of our external shock model. 


\section{Fitting Results}

\subsection{Constraints on Parameters}

Based on the analytical model we mentioned above, our procedure of further narrowing down the physical parameter space in the self-similar phase includes the following steps:

In the wind environment:

(1) According to the expression of $F_{\nu} \propto t^{-\alpha} \nu^{-\beta}$ in different spectral regimes given by Wu et al. (2005) and the observed high-energy, X-ray and optical temporal and spectral indices, $(p, \varepsilon)$ can be roughly estimated (see Tab. 1).

(2) For simplicity, $\varepsilon \approx \epsilon_{e}$ in the fast cooling regime and then $\varepsilon$ decays slowly (where we denote $t_{c m}$ as the time when $\nu_{c}=\nu_{m}$, and obtain that $\varepsilon$ decays as $\varepsilon \sim \epsilon_{e}\left(t / t_{c m}\right)^{2-p}$ in the slow cooling regime (i.e., setting $j \equiv-m /(2 m+2)$, we have, after $t_{c m}, \Gamma \propto t^{j}$, then $\nu_{m} \propto t^{-(j+1)}$ and $\nu_{c} \propto t^{(j+1)}$, and equation (5) provides $\varepsilon \propto \epsilon_{e}\left(\nu_{m} / \nu_{c}\right)^{(p-2) / 2} \propto t^{(2-p)(j+1)}$, as long as $\varepsilon \leq 2 / 3, j+1 \sim 2 / 3)$.

(3) Because of the definition for the deceleration time [set $k=2$ in Equations (1) and

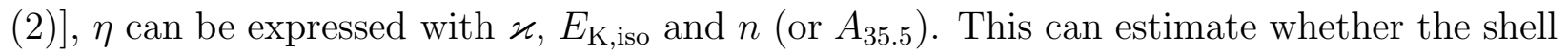
thickness is thin enough to neglect the reverse shock's contribution to the total flux density.

(4) Hereafter the free parameters are $\varkappa, E_{\mathrm{K} \text {,iso }}, \epsilon_{\mathrm{B}}$, and $n$ (or $A_{35.5}$ ). The observed flux density in different wavebands (radio, optical, X-ray, $>100 \mathrm{MeV}$ ) can be combined and eventually simultaneously be solved. In this paper, the temporal indices of the X-ray and optical light curves concurrently evolve according to equation (10) and thus $\epsilon_{B}$ can therefore be expressed with $\varkappa, E_{\mathrm{K} \text {,iso }}$ and $n$ (or $A_{35.5}$ ).

(5) To justify whether the early high energy LAT photons come from the same region as the late afterglow, what we need to do is to replace $\epsilon_{B}$ in the form of $\varkappa, E_{\mathrm{K} \text {,iso }}$ and $n$ (or $A$ ) from step (4). To confirm whether SSC make a significant contribution to the GeV afterglow or Klein-Nishina effect changes the synchrotron and SSC spectra, we need to discuss the value of $Y\left(\gamma_{c}\right)$ in the slow cooling regime or $Y\left(\gamma_{m}\right)$ in the fast cooling regime (Nakar et al. 2009), therefore $Y\left(\gamma_{G e V}\right)$ can be estimated (Wang et al. 2010). If no solution is found in the range of $\left(10^{53} \mathrm{ergs} \precsim E_{\mathrm{K} \text {,iso }} \precsim 10^{56} \mathrm{ergs}\right.$ and $\left.10^{-2} \precsim A_{35.5} \precsim 10^{2}\right)$, the $>100 \mathrm{MeV}$ photons may come from the other component, vise vesa.

(6) From discussions about the parameter space of $\left(A_{35.5}\right.$ and $\varkappa$ ) according to observational statistics, one can obtain several best fittings. In addition, The ratio $\xi$ of the wind and homogeneous medium density, $\epsilon_{e}$ and $\epsilon_{B}$ at early and late times may have slight changes, which can also be legally adjusted in practise. 


\subsection{Best Fittings}

We put the above procedure into operation, and best fitting parameters are shown in Tab. 2. The evolution of characteristic frequencies for each burst is expected as follows. Here our LAT data are taken from Zhang et al. (2010) and X-ray data were reported by Swift (Evans et al. 2007, 2009).

From the lightcurves, we found that from $t_{d e c}$ on, $\alpha_{G e V, X, o p t}>0, \beta_{G e V, X, o p t}>0$. According to the analytical expression from Wu et al. (2005), before the blast wave reaches the density jump, GeV photons at early decaying time (e.g., $t_{d e c} \leq t=t_{I} \leq 10^{3}$ s) has two possibilities:

$$
F_{s y n, G e V} \propto \begin{cases}t^{-\frac{3 p-2-(p-2) \varepsilon}{2(2-\varepsilon)} \nu_{G e V}^{\frac{-p}{2}}}, & \nu_{G e V} \geq \nu_{m}(t) \geq \nu_{c}(t) \\ t^{-\frac{3 p-2-(p-2) \varepsilon}{2(2-\varepsilon)}+\frac{p-2}{4-p}} \nu_{G e V}^{\frac{-p}{2}}, & \nu_{G e V} \geq \nu_{c}(t) \geq \nu_{m}(t)\end{cases}
$$

while the lower energy bands (X-ray, optical) at $t=t_{I I}>10^{4} \mathrm{~s}$, may lie in one of the following slow cooling cases:

$$
\begin{array}{ll}
F_{o p t} \propto t^{-\frac{3 p-1-(p-1) \varepsilon}{2(2-\varepsilon)}} \nu_{o p t}^{\frac{-(p-1)}{2}}, F_{X} \propto t^{-\frac{3 p-1-(p-1) \varepsilon}{2(2-\varepsilon)} \nu_{X}^{\frac{-(p-1)}{2}},} & \nu_{c}(t) \geq \nu_{X} \geq \nu_{o p t} \geq \nu_{m}(t), \\
F_{o p t} \propto t^{-\frac{3 p-1-(p-1) \varepsilon}{2(2-\varepsilon)}} \nu_{o p t}^{\frac{-(p-1)}{2}}, F_{X} \propto t^{-\frac{3 p-2-(p-2) \varepsilon}{2(2-\varepsilon)}+\frac{p-2}{4-p}} \nu_{X}^{\frac{-p}{2}}, & \nu_{X} \geq \nu_{c}(t) \geq \nu_{o p t} \geq \nu_{m}(t), \\
F_{o p t} \propto t^{-\frac{3 p-2-(p-2) \varepsilon}{2(2-\varepsilon)}+\frac{p-2}{4-p}} \nu_{o p t}^{\frac{-p}{2}}, F_{X} \propto t^{-\frac{3 p-2-(p-2) \varepsilon}{2(2-\varepsilon)}+\frac{p-2}{4-p}} \nu_{X}^{\frac{-p}{2}}, & \nu_{X} \geq \nu_{o p t} \geq \nu_{c}(t) \geq \nu_{m}(t) .
\end{array}
$$

for $090902 \mathrm{~B}$ radio band, at $t=t_{I I I}$ when $\beta_{\text {radio }}>0$ (i.e. $\nu_{\text {radio }} \geq \nu_{m}$ ), it is similar to the optical spectrum and lightcurve. While $\beta_{\text {radio }}<0$,

$$
F_{\text {radio }} \propto t^{-\frac{\varepsilon}{3(2-\varepsilon)}} \nu_{\text {radio }}^{\frac{1}{3}}, \quad \nu_{m}(t) \geq \nu_{\text {radio }}
$$

\subsubsection{GRB080916C}

Our optical photometry of this burst is taken from Greiner et al. (2009). First of all, if the X-ray and optical afterglow shares the same source as the high-energy photons detected by LAT, their spectral indices $\beta_{o b, x}=0.50 \pm 0.16$ and $\beta_{o b, o p t}=0.38 \pm 0.02$ should be consistent with the $\mathrm{GeV}$ photons, $\alpha_{o b, L A T}=1.33 \pm 0.08$ and $\beta_{o b, L A T}=1.1 \pm 0.1$. This is preliminarily valid only in the case of $\nu_{G e V} \geq \max \left[\nu_{c}\left(t_{I}\right), \nu_{m}\left(t_{I}\right)\right]$ and $\nu_{c}\left(t_{I I}\right) \geq \nu_{X} \geq \nu_{\text {opt }} \geq \nu_{m}\left(t_{I I}\right)$, when $2.0 \leq p \leq 2.32$ and $0<\epsilon_{e} \leq 0.48$.

Consequently, the peak of the LAT light curve lying at $t_{d e c} \sim 6 \mathrm{~s}$ can be considered as the deceleration time of this burst. Therefore, equations (11) and (2) lead to the relationship 
among the isotropic kinetic energy $E_{\mathrm{K}, 55}$, the bulk Lorentz factor $\eta_{3}$, and the wind parameter $A_{35.5}$ at the very moment: $\eta_{3} \sim 0.40 E_{\mathrm{K}, 55}^{1 / 4} A_{35.5}^{-1 / 4}(1+2 \varkappa)^{1 / 4}$.

Equation (3) provides, at $t \leq t_{d e c}, f / \Gamma^{2}=72 \exp (5.37 \varkappa)(1+\varkappa)(1+2 \varkappa)^{-1} t^{-3 \varkappa-2}>1$. Thus the reverse shock is nearly Newtonian.

Subsequently, when the blast wave is embedded in the wind bubble, we assume that the typical value $\varepsilon\left(t_{I}\right) \sim 1 / 3$, while $\varepsilon\left(t_{I I}\right) \sim 0.0$, and the characteristic frequencies evolve as

$$
\begin{array}{r}
\nu_{c}\left(t_{I}\right)=1.21 \times 10^{9} \mathrm{~Hz} E_{\mathrm{K}, 55}^{1 / 2} A_{35.5}^{-2} \epsilon_{B, 0}^{-3 / 2}(1+2 \varkappa)^{-5 / 2} t_{I}{ }^{2 / 5}\left[1+Y\left(\gamma_{c}, t_{I}\right)\right]^{-2}, \\
\nu_{c}\left(t_{I I}\right)=1.03 \times 10^{9} \mathrm{~Hz} E_{\mathrm{K}, 55}^{1 / 2} A_{35.5}^{-2} \epsilon_{B, 0}^{-3 / 2}(1+2 \varkappa)^{-5 / 2} t_{I I}{ }^{1 / 2}\left[1+Y\left(\gamma_{c}, t_{I I}\right)\right]^{-2}, \\
\nu_{m}\left(t_{I}\right)=8.66 \times 10^{23} \mathrm{~Hz}_{\mathrm{K}, 55}^{1 / 2} \epsilon_{e, 0}^{2} \epsilon_{B, 0}^{1 / 2}(1+2 \varkappa)^{3 / 2} t_{I}{ }^{-8 / 5} f_{p}{ }^{2}, \\
\nu_{m}\left(t_{I I}\right)=7.39 \times 10^{23} \mathrm{~Hz} E_{\mathrm{K}, 55}^{1 / 2} \epsilon_{e, 0}^{2} \epsilon_{B, 0}^{1 / 2}(1+2 \varkappa)^{3 / 2} t_{I I}{ }^{-3 / 2} f_{p}{ }^{2} .
\end{array}
$$

Setting $p=2.3$ and $\epsilon_{e, 0}=1 / 3$ as a trail, we only consider the synchrotron emissivity,

$$
F_{\text {syn }, \mathrm{GeV}}(13 \mathrm{~s})=18.5 \mu \mathrm{Jy} E_{\mathrm{K}, 55}^{43 / 40} \epsilon_{B, 0}^{3 / 40}(1+2 \varkappa)^{9 / 40}\left[1+Y\left(\gamma_{c}, 13 \mathrm{~s}\right)\right]^{-1} .
$$

We found that equation (16) is approximately the same under both fast cooling and slow cooling condition, so do with the following equation (24) and (34).

$$
F_{\text {syn }, X}\left(1.01 \times 10^{5} \mathrm{~s}\right)=1.76 \times 10^{5} \mu \mathrm{Jy} E_{\mathrm{K}, 55}^{33 / 40} A_{35.5} \epsilon_{B, 0}^{33 / 40}(1+2 \varkappa)^{59 / 40} \sim 7.73 \times 10^{-2} \mu \mathrm{Jy} .
$$

From equation (17), we have $\epsilon_{B, 0}=1.96 \times 10^{-8} E_{\mathrm{K}, 55}^{-1} A_{35.5}^{-40 / 33}(1+2 \varkappa)^{-9 / 5}$, so $t_{c m} \leq 13 \mathrm{~s}$ in the case that $10^{-2} \precsim A_{35.5}$. Therefore, $\nu_{m}(13 \mathrm{~s})<\nu_{\mathrm{c}}(13 \mathrm{~s})$ and $F_{\text {syn }, \mathrm{GeV}}(13 \mathrm{~s}) \leq 0.83 \mu \mathrm{Jy}$, which yields

$$
5.83 E_{\mathrm{K}, 55} A_{35.5}^{-1 / 10}(1+2 \varkappa)^{1 / 10} \leq 1+Y\left(\gamma_{c}, 13 \mathrm{~s}\right)<54 E_{\mathrm{K}, 55} A_{35.5}^{1 / 5}(1+2 \varkappa)^{-1 / 5}
$$

From Wang et al. (2010), we have, in the wind, if $t_{I}>13 \mathrm{~s}, Y\left(\gamma_{c}, t_{I}\right)<Y\left(\gamma_{c}, 13 \mathrm{~s}\right)$. This have been shown in Fig. 4.c. Hence, as long as $A_{35.5}(1+2 \varkappa)^{-1} t_{I}<20.2$ (e.g. $10^{-2} \precsim A_{35.5}(1+$ $2 \varkappa)^{-1}<1.56$ at $13 \mathrm{~s}$ or $10^{-2} \precsim A_{35.5}(1+2 \varkappa)^{-1} \sim 0.02$ at $\left.10^{3} \mathrm{~s}\right)$,

$\widehat{\frac{\gamma_{m}\left(t_{I}\right)}{\gamma_{c}\left(t_{I}\right)}}=3.0 \times 10^{-3} E_{\mathrm{K}, 55}^{-1} A_{35.5}^{2 / 5} t_{I}^{3 / 5}(1+2 \varkappa)^{-2 / 5}\left[1+Y\left(\gamma_{c}, t_{I}\right)\right]<0.18 A_{35.5}^{3 / 5} t_{I}^{3 / 5}(1+2 \varkappa)^{-3 / 5}<1$,

According to Nakar et al. (2009), in the slow cooling, equation (19) leads to $Y\left(\gamma_{c}, t_{I}\right)<$ 1 , then SSC cooling has no effect on the electron distribution. From the analytical solution of Wang et al. (2010), $Y\left(\gamma_{G e V}, t_{I}\right) \ll 1$ can be estimated. With the assumption that the $\mathrm{GeV}$ luminosity is dominated by synchrotron emission, $F_{\text {syn }, \mathrm{GeV}}(13 \mathrm{~s}) \sim 0.83 \mu \mathrm{Jy}$ provide a stronger 
constraint, $E_{\mathrm{K}, 55}=0.19 A_{35.5}^{1 / 10}(1+2 \varkappa)^{-1 / 10}$.In such parameter space, we have $\tau_{\gamma \gamma} \ll 1$, so the absorption does not contribute to the early rising.

All the above relations show that $\epsilon_{e, 0}, E_{K, 55}$ and $A_{35.5}$ can be expressed with $\eta_{3}$ and $\varkappa$, the physical mechanism for the rising part of the lightcurve can be considered as contribution of the structured ejecta. According to observational statistics, the best fitting yields $E_{\mathrm{K}, 55}=$ 0.13, $A_{35.5}=0.02, \epsilon_{e, 0}=0.3, \epsilon_{B, 0}=5.81 \times 10^{-6}$, and $\eta_{3}=0.74$. As the result shown in Fig. fig:080916C: before the deceleration time, the bulk Lorentz factor of the swept blast wave increases with $\varkappa=0.5$ (which is self-consistent with our assumption for $\varkappa$ and thin shell case), $t_{c m}$ occurs at $\sim 4$.5s then electrons are slow cooling, and deceleration begins from $\sim 6 \mathrm{~s}$, the density jump is expected around $10^{5.3}-10^{5.6} \mathrm{~s}$ (i.e., $3.25 \times 10^{18} \mathrm{~cm}$ ). After breaking out to the interstellar medium, the surrounding density is around $1.17 \times 10^{-3} \mathrm{~cm}^{-3}$.

\subsubsection{GRB090902B}

Along with the LAT and XRT data mentioned above, our optical photometry here is taken from Pandey et al. (2010), as well as the 8.5GHz data reported by the VLA (van der Horst et al. 2009). With the assumption that the late X-ray, optical and radio afterglow and the early LAT photons originate from the same region, their spectral indices $\beta_{o b, x}=0.90 \pm 0.13$ and $\beta_{o b, o p t}=0.76 \pm 0.07$ should be consistent with the GeV photons, $\alpha_{o b, L A T}=1.4 \pm 0.06$. The same as the above analysis, $\nu_{G e V} \geq \max \left[\nu_{c}\left(t_{I}\right), \nu_{m}\left(t_{I}\right)\right]$ and $\nu_{c}\left(t_{I I}\right) \geq \nu_{X} \geq \nu_{o p t} \geq \nu_{m}\left(t_{I I}\right)$, respectively, which result in the plausible light curve and spectrum similar to the above case. Therefore, roughly speaking, $2.3 \leq p \leq 2.6$ and $0.17 \leq \epsilon_{e} \leq 0.43$ are the available region.

Although the peculiar "soft-hard" spectral evolution seems tricky in the prompt emission phase, its duration is so short $\left(T_{0}-T_{0}+10 \mathrm{~s}\right)$ and it ends before the deceleration time (Zhang et al. 2010), which, is understandable in the frame of structured ejecta acceleration (we will discuss it in Sec 5 ), so the external shock model is still applicable. In addition, although a $33 \mathrm{GeV}$ photon was detected at $82 \mathrm{~s}$ after the trigger, we consider the $11.2 \mathrm{GeV}$ photon at $\sim 10 \mathrm{~s}$ as the highest energy photon of estimation for the lower Lorentz factor because the arrival time of the $33 \mathrm{GeV}$ photon is far beyond the prompt emission phase and may not merely be attributed from the external shock but some other origins.

The turning point of the LAT light curve occurs at $\sim 7 \mathrm{~s}$. This indicates that a relationship among $E_{\mathrm{K}, 55}, \eta_{3}$, and $A_{35.5}$ becomes $\eta_{3} \sim 0.33 E_{\mathrm{K}, 55}^{1 / 4} A_{35.5}^{-1 / 4}(1+2 \varkappa)^{1 / 4}$. Hence, equation (3) yields that at $t \leq t_{d e c}$ and $f / \Gamma^{2}=98 \exp (5.94 \varkappa)(1+\varkappa)(1+2 \varkappa)^{-1} t^{-3 \varkappa-2}>1$, so the thin shell case is applicable in this fitting.

Similar to analytical solutions of GRB080916C we mentioned above, characteristic fre- 
quencies of the photons observed by LAT and later multiband (X-ray, optical, radio) afterglow can be estimated. Additionally, when we come to the radio band, SSA may be crucial.

Here again, we assume that the typical value $\varepsilon\left(t_{I}\right) \sim 1 / 3$, while $\varepsilon\left(t_{I I}\right) \sim 0.0$, with model parameters $\left(E_{\mathrm{K}, 55}, \epsilon_{B, 0}, \epsilon_{e, 0}\right.$, and $\left.A_{35.5}\right)$, the characteristic frequencies evolve as

$$
\begin{array}{r}
\nu_{c}\left(t_{I}\right)=3.29 \times 10^{9} \mathrm{~Hz} E_{\mathrm{K}, 55}^{1 / 2} A_{35.5}^{-2} \epsilon_{B, 0}^{-3 / 2}(1+2 \varkappa)^{-5 / 2} t_{I}{ }^{2 / 5}\left[1+Y\left(\gamma_{c}, t_{I}\right)\right]^{-2}, \\
\nu_{c}\left(t_{I I}\right)=2.71 \times 10^{9} \mathrm{~Hz} E_{\mathrm{K}, 55}^{1 / 2} A_{35.5}^{-2} \epsilon_{B, 0}^{-3 / 2}(1+2 \varkappa)^{-5 / 2} t_{I I}{ }^{1 / 2}\left[1+Y\left(\gamma_{c}, t_{I I}\right)\right]^{-2}, \\
\nu_{m}\left(t_{I}\right)=6.52 \times 10^{23} \mathrm{~Hz}_{\mathrm{K}, 55}^{1 / 2} \epsilon_{e, 0}^{2} \epsilon_{B, 0}^{1 / 2}(1+2 \varkappa)^{3 / 2} t_{I}{ }^{-8 / 5} f_{p}{ }^{2}, \\
\nu_{m}\left(t_{I I}\right)=5.37 \times 10^{23} \mathrm{~Hz} E_{\mathrm{K}, 55}^{1 / 2} \epsilon_{e, 0}^{2} \epsilon_{B, 0}^{1 / 2}(1+2 \varkappa)^{3 / 2} t_{I I}{ }^{-3 / 2} f_{p}{ }^{2} .
\end{array}
$$

Setting $p=2.4, \epsilon_{e, 0}=0.4$ as a trail, the synchrotron emissivity is given by

$$
\begin{gathered}
F_{\text {syn }, \mathrm{GeV}}(10 \mathrm{~s})=14.58 \mu \mathrm{JyE}_{\mathrm{K}, 55}^{11 / 10} \epsilon_{\mathrm{B}, 0}^{1 / 10}(1+2 \varkappa)^{3 / 10}\left[1+\mathrm{Y}\left(\gamma_{\mathrm{c}}, 10 \mathrm{~s}\right)\right]^{-1} . \\
F_{\text {syn }, X}\left(1.17 \times 10^{5} \mathrm{~s}\right)=6.72 \times 10^{5} \mu \mathrm{JyE}_{\mathrm{K}, 55}^{17 / 20} \mathrm{~A}_{35.5} \epsilon_{\mathrm{B}, 0}^{17 / 20}(1+2 \varkappa)^{31 / 20} \sim 0.18 \mu \mathrm{Jy}
\end{gathered}
$$

From equation (25) , we have $\epsilon_{B, 0}=1.87 \times 10^{-8} E_{\mathrm{K}, 55}^{-1} A_{35.5}^{-20 / 17}(1+2 \varkappa)^{-9 / 5}$, so $t_{c m} \leq 10 \mathrm{~s}$ in the case that $A_{35.5} \geq 10^{-2}$. Therefore, $\nu_{m}(10 \mathrm{~s})<\nu_{\mathrm{c}}(10 \mathrm{~s})$ and $F_{\text {syn }, \mathrm{GeV}}(10 \mathrm{~s}) \leq 1.59 \mu \mathrm{Jy}$, which yields,

$$
1.54 E_{\mathrm{K}, 55} A_{35.5}^{-3 / 25}(1+2 \varkappa)^{3 / 25} \leq 1+Y\left(\gamma_{c}, 10 \mathrm{~s}\right)<55 \mathrm{E}_{\mathrm{K}, 55} \mathrm{~A}_{35.5}^{9 / 50}(1+2 \varkappa)^{-9 / 50}
$$

Meanwhile, for the radio band at $t_{I I I} \mathrm{~s}$, because of the light curve index $\sim 1 / 3$, SSA can be neglected, we have

$$
\begin{array}{r}
\nu_{m}\left(5.23 \times 10^{5} \mathrm{~s}\right)=9.14 \times 10^{10} \mathrm{~A}_{35.5}^{-10 / 17}(1+2 \varkappa)^{10 / 17}>8.5 \mathrm{GHz} \\
F_{\text {syn }, 8.5 \mathrm{GHz}}\left(5.23 \times 10^{5} \mathrm{~s}\right)=1.89 \times 10^{2} \mu \mathrm{JyA}_{35.5}^{3 / 5}(1+2 \varkappa)^{-3 / 5} \sim 56_{-26}^{+24} \mu \mathrm{Jy} .
\end{array}
$$

Therefore, it should be $0.05<A_{35.5}(1+2 \varkappa)^{-1}<0.24$, and if $A_{35.5}(1+2 \varkappa)^{-1} t_{I}<46.7$ (e.g. $A_{35.5}(1+2 \varkappa)^{-1}<0.24$ at 10 s or $0.05<A_{35.5}(1+2 \varkappa)^{-1} \sim 0.05$ at $10^{3} \mathrm{~s}$ ),

$\widehat{\frac{\gamma_{m}\left(t_{I}\right)}{\gamma_{c}\left(t_{I}\right)}}=1.8 \times 10^{-3} E_{\mathrm{K}, 55}^{-1} A_{35.5}^{2 / 5}(1+2 \varkappa)^{-2 / 5} t^{3 / 5}\left[1+Y\left(\gamma_{c}, t_{I}\right)\right]<0.10 A_{35.5}^{3 / 5}(1+2 \varkappa)^{-3 / 5} t_{I}^{3 / 5}<1$.

Under such a parameter space, equation (29) leads to $Y_{c, t_{I}}<1$, SSC makes a little effect because of the Klein-Nishina suppression, so the estimation that $Y\left(\gamma_{G e V}, t_{I}\right) \ll 1$ is self-consistent. With the assumption that the GeV luminosity is dominated by synchrotron 
emission, $F_{\text {syn }, \mathrm{GeV}}(10 \mathrm{~s}) \sim 1.59 \mu \mathrm{Jy}$ provide a stronger constraint, $E_{\mathrm{K}, 55}=0.65 A_{35.5}^{2 / 17}(1+$ $2 \varkappa)^{-2 / 17}$ and $\tau_{\gamma \gamma} \ll 1$.

Using the above relations, we can express $\epsilon_{e, 0}, E_{K, 55}$ and $A_{35.5}$ by $\eta_{3}$ and $\varkappa$, and fit the data according to observational statistic. The best fitting requires $E_{\mathrm{K}, 55}=0.44, A_{35.5}=0.06$, $\epsilon_{e, 0}=0.4, \epsilon_{B, 0}=4.56 \times 10^{-7}$, and $\eta_{3}=0.60$. Fig. fig:parameter shows the circumburst density, radius and characteristic frequency, and Compton $Y_{\mathrm{GeV}}$ and $Y_{\gamma_{c}}$ factor evolution under such a parameter space. Fig. fig:090902B shows the spectrum and light curve fitting. Before the deceleration time $(7 \mathrm{~s})$, the bulk Lorentz factor of the blast wave increases with a slope of $\varkappa=0.8$ (which is self-consistent with $\varkappa$ and thin shell case). The shock-accelerated electrons are cooling fast early before $\sim 0.72 \mathrm{~s}$, the density jump is estimated around $10^{5.1}-$ $10^{6} \mathrm{~s}$ (i.e., at radius of $4.48 \times 10^{18} \mathrm{~cm}$ ). After breaking out to the interstellar medium, the surrounding density is as low as $1.91 \times 10^{-3} \mathrm{~cm}^{-3}$. These parameters are consistent with the radio light curve except for the first data and is reliable when the jet break takes place nearly at the same time. We find that the jet angle is $0.04 \mathrm{rad}$ and collimation-corrected energy is $E_{\text {jet }} \sim 1.51 \times 10^{51} \mathrm{ergs}$.

\subsubsection{GRB090926A}

$\mathrm{V}$ band and $\mathrm{R}$ band data of this burst are taken from Swenson et al. (2010) and Rau et al. (2010), respectively. Our analysis procedure is the same as mentioned above. Due to the hypothesis of the same source, the temporal index of the LAT photons $\alpha_{o b, L A T}=$ $2.05 \pm 0.14$, should be consistent with the spectral indices $\beta_{o b, x}=1.12 \pm 0.13$ and $\beta_{o b, o p t}=$ $1.03 \pm 0.05$. This leads to a fairly broad plausible range for $3 \geq p \geq 2$ and $\epsilon_{e} \sim 10^{-1}$ (e.g.under the condition of $\nu_{G e V}>\max \left[\nu_{m}\left(t_{I}\right), \nu_{c}\left(t_{I}\right)\right], \nu_{c}\left(t_{I I}\right)>\nu_{R, V}$ requires for $3 \geq p \geq 2.96$ and $0.62>\varepsilon>0.37$, while $2.5 \geq p \geq 2$ and $0.70>\varepsilon>0.45$ as long as $\left.\nu_{c}\left(t_{I I}\right)<\nu_{X}\right)$. However, since the light curve after the density jump is a little bit steeper than the early part, $\varepsilon$ (the latter the smaller) should not deviate too much from the typical value (i.e., 1/3, otherwise, the decrease of loss efficiency will cause the decaying slope much shallower), whereas the decay of LAT is steep, which requires a large $p$ (i.e., 3.0). Besides, in the afterglow phase, we can reasonably believe that the first flare at $70-95 \mathrm{ks}$ comes from an interaction of the blast wave and density jump. The second "flare" may come from late energy injection or another density jump, and in this paper, we smooth it as the simple power law.

Because the deceleration time is estimated as $\sim 16 \mathrm{~s}, \eta_{3}$ can be written by $\eta_{3} \sim$ $0.27 E_{\mathrm{K}, 55}^{1 / 4} A_{35.5}^{-1 / 4}(1+2 \varkappa)^{1 / 4}$. Hence, at $t \leq t_{d e c}, f / \Gamma^{2}=512 \exp (8.32 \varkappa)(1+\varkappa)(1+2 \varkappa)^{-1} t^{-3 \varkappa-2}>$ 1 , so the reverse shock is negligible in this fitting. With the typical value $\varepsilon\left(t_{I}\right) \sim 1 / 3$, while 
$\varepsilon\left(t_{I I}\right) \sim 0.0$, The characteristic frequencies evolve as

$$
\begin{array}{r}
\nu_{c}\left(t_{I}\right)=3.09 \times 10^{9} \mathrm{~Hz} E_{\mathrm{K}, 55}^{1 / 2} A_{35.5}^{-2} \epsilon_{B, 0}^{-3 / 2}(1+2 \varkappa)^{-5 / 2} t_{I}{ }^{2 / 5}\left[1+Y\left(\gamma_{c}, t_{I}\right)\right]^{-2}, \\
\nu_{c}\left(t_{I I}\right)=2.34 \times 10^{9} \mathrm{~Hz} E_{\mathrm{K}, 55}^{1 / 2} A_{35.5}^{-2} \epsilon_{B, 0}^{-3 / 2}(1+2 \varkappa)^{-5 / 2} t_{I I}{ }^{1 / 2}\left[1+Y\left(\gamma_{c}, t_{I I}\right)\right]^{-2}, \\
\nu_{m}\left(t_{I}\right)=7.43 \times 10^{23} \mathrm{~Hz} E_{\mathrm{K}, 55}^{1 / 2} \epsilon_{e, 0}^{2} \epsilon_{B, 0}^{1 / 2}(1+2 \varkappa)^{3 / 2} t_{I}{ }^{-8 / 5} f_{p}{ }^{2}, \\
\nu_{m}\left(t_{I I}\right)=5.63 \times 10^{23} \mathrm{~Hz}_{\mathrm{K}, 55}^{1 / 2} \epsilon_{e, 0}^{2} \epsilon_{B, 0}^{1 / 2}(1+2 \varkappa)^{3 / 2} t_{I I}{ }^{-3 / 2} f_{p}{ }^{2} .
\end{array}
$$

Setting $p=3.0, \epsilon_{e, 0}=0.4$ as a trail, the synchrotron emissivity is calculated by

$$
\begin{gathered}
F_{\text {syn }, \mathrm{GeV}}(36 \mathrm{~s})=28.7 \mu \mathrm{JyE}_{\mathrm{K}, 55}^{5 / 4} \epsilon_{\mathrm{B}, 0}^{1 / 4}(1+2 \varkappa)^{3 / 4}\left[1+\mathrm{Y}\left(\gamma_{\mathrm{c}}, 36 \mathrm{~s}\right)\right]^{-1}, \\
F_{s y n, X}\left(5.3 \times 10^{4} \mathrm{~s}\right)=1.42 \times 10^{6} \mu \mathrm{JyE}_{\mathrm{K}, 55} \mathrm{~A}_{35.5} \epsilon_{\mathrm{B}, 0}(1+2 \varkappa)^{2} \sim 0.33 \mu \mathrm{Jy} .
\end{gathered}
$$

From equation (35), we have $\epsilon_{B, 0}=2.32 \times 10^{-7} E_{\mathrm{K}, 55}^{-1} A_{35.5}^{-1}(1+2 \varkappa)^{-2}$, so $t_{c m}=4.31 / E_{\mathrm{K}, 55} \leq$ $36 \mathrm{~s}$ in the case that $E_{K, 55} \geq 0.12$. Therefore, $\nu_{m}(36 \mathrm{~s})<\nu_{\mathrm{c}}(36 \mathrm{~s})$ and $F_{s y n, \mathrm{GeV}}(36 \mathrm{~s}) \leq 0.37 \mu \mathrm{Jy}$, which yields,

$$
1.71 E_{\mathrm{K}, 55} A_{35.5}^{-1 / 4}(1+2 \varkappa)^{1 / 4} \leq 1+Y\left(\gamma_{c}, 36 \mathrm{~s}\right)<8.38 \mathrm{E}_{\mathrm{K}, 55} .
$$

Hence,as long as $A_{35.5}^{1 / 2}(1+2 \varkappa)^{-1 / 2} t_{I}^{3 / 5}<65.8$ (e.g., $A_{35.5}(1+2 \varkappa)^{-1}<58.7$ at 36 s or $A_{35.5}(1+2 \varkappa)^{-1}<1$ at $\left.10^{3} \mathrm{~s}\right)$,

$\widehat{\frac{\gamma_{m}\left(t_{I}\right)}{\gamma_{c}\left(t_{I}\right)}}=1.8 \times 10^{-3} E_{\mathrm{K}, 55}^{-1} A_{35.5}^{1 / 2}(1+2 \varkappa)^{-1 / 2} t^{3 / 5}\left[1+Y\left(\gamma_{c}, t_{I}\right)\right]<0.015 A_{35.5}^{1 / 2}(1+2 \varkappa)^{-1 / 2} t_{I}^{3 / 5}<1$.

In the slow cooling case, equation (37) leads to $Y\left(\gamma_{c}, t_{I}\right)<1$, SSC makes a little effect due to the Klein-Nishina suppression, because the estimation of $Y\left(\gamma_{\mathrm{GeV}}, t_{I}\right) \ll 1$ is selfconsistent.

Meanwhile, during $1 \leq t_{I} \leq 10^{2.5} \mathrm{~s}$, in the case of fast cooling $\left(E_{55}<0.12\right)$ that $\nu_{m}(36 \mathrm{~s})>\nu_{\mathrm{c}}(36 \mathrm{~s})$ and $F_{s y n, \mathrm{GeV}}(36 \mathrm{~s}) \leq 0.37 \mu \mathrm{Jy}, A_{35.5}(1+2 \varkappa)^{1 / 5} \leq 0.026$,

$$
\frac{\gamma_{m}\left(t_{I}\right)}{\widehat{\gamma_{m}\left(t_{I}\right)}}=2.38 \times 10^{3} A_{35.5}^{-1 / 2}(1+2 \varkappa)^{1 / 2} t_{I}^{-8 / 5}>1
$$

According to Nakar et al. (2009), this is within the strong KN regime (case II). Analytical discussion with the above confines yield to the possibilities of case IIb and IIc, both leads to $Y\left(\gamma_{G e V}, t_{I}\right) \ll 1$. Hence, the contribution from SSC could be neglected, too. In both fast and slow cooling, $F_{\text {syn }, \mathrm{GeV}}(36 \mathrm{~s}) \sim 0.37 \mu \mathrm{Jy}$ provide a stronger constraint, $E_{\mathrm{K}, 55}=0.70 A_{35.5}^{1 / 4}(1+$ $2 \varkappa)^{-1 / 4}$ and $\tau_{\gamma \gamma} \ll 1$. 
Using the above relations, we can express $\epsilon_{e, 0}, E_{K, 55}$ and $A_{35.5}$ by $\eta_{3}$ and $\varkappa$, and fit the data according to observational statistic. When $E_{\mathrm{K}, 55}=0.43, A_{35.5}=0.03, \epsilon_{e, 0}=0.35$,

$\epsilon_{B, 0}=8.18 \times 10^{-6}$, and $\eta_{3}=0.56$, fitting seems plausible (Fig. fig:090926A). Before the deceleration time, the Lorentz factor of the blast wave increases with a slope of $\varkappa=0.3$, $t_{c m} \sim 8.8 \mathrm{~s}$ and from $\sim 16 \mathrm{~s}$ significant deceleration appears. The blast wave enters the ISM around $10^{4.8}-10^{4.9} \mathrm{~s}$ (i.e., at radius of $4.41 \times 10^{18} \mathrm{~cm}$ ). The medium density surrounding the bubble is $1.3 \times 10^{-3} \mathrm{~cm}^{-3}$.

\subsection{Analysis of Results}

Tab. 2 shows all the best fit parameters for the above three GRBs. Common results can be found:

(1) In all the three burst fittings, the thin shell case is applicable, the very beginning of $\mathrm{GeV}$ flux densities are forward-shock dominated. The rising slope comes from the contribution of the power-law increasing bulk Lorentz factor of structured ejecta. These are self-consistent with the thin shell case, indicating that the central engine is very likely to be the core collapse of a massive star (we will discuss it in Sec 5 ). Our model implies that the central object would keep on accreting matter even after the LAT trigger.

(2) The remaining isotropic kinetic energy of the fireball after the prompt emission are all $\sim 10^{54}$ ergs, indicating $\sim 30 \%-90 \%$ of the total energy has been emitted in the prompt emission. This is because $t_{c m}$ occurs before the deceleration time for all the three GRBs and the following dynamics could be considered as being quasi-adiabatic. As a matter of fact, $\epsilon_{e}$ in our sample fittings is $\leqslant 2 / 3$, which meets the requirement of radiation efficiency related to the analytic procedure from $\mathrm{Wu}$ et al. (2005). Practically, slow cooling of electrons starts before the deceleration time, and a jet break appears much later, so Wu's spherical-like solution is tenable in this piece. A low value of $\epsilon_{B}\left(\sim 10^{-7}-10^{-5}\right)$ indicates a weak magnetic field, especially for GRB 080916C and GRB 090902B. These GRBs have been studied by Kumar \& Barniol Duran (2009), whose inferred magnetic field (along with several other LAT-detected events) is consistent with shock compression of a modest circumstellar field ( $B \succsim 30 \mu \mathrm{G})$. That is to say, no dynamo process is necessary to generate the magnetic field needed for the observed synchrotron afterglow emission. In our best fittings, we find $B>380 \mu \mathrm{G}$ for GRB080916C and $B>106 \mu \mathrm{G}$ for GRB090902B even when $T_{0}+10^{7} \mathrm{~s}$, being broadly consistent with the results of Piran \& Nakar (2010) and Li (2010). Meanwhile, when the blast wave enters the constant-density circumburst medium, the preshock magnetic field can be calculated with our fitting parameters as $B=\left(2 \pi m_{p} \epsilon_{B} n\right)^{1 / 2} \approx 8.0 \mu \mathrm{G}$ for $080916 \mathrm{C}$ and $2.6 \mu \mathrm{G}$ for $090902 \mathrm{~B}$, that suggests no magnetic field amplification. 
(3) Although the ratio of $\epsilon_{e}$ and $\epsilon_{B}$ is large, synchrotron self-Compton is not significantly contributed to the observed flux density in the $\mathrm{GeV}$ band because of the Klein-Nishina suppression effect. In our sample fittings, only synchrotron radiation from the external forward shock is enough to contribute to the high energy emission and the synchrotron selfCompton component can be neglected because $Y\left(\gamma_{G e V}\right) \ll 1$ in this piece. In particular, for GRB 090902B, from our best-fitting parameters, the maximum electron Lorentz factor at 82 s indicates the highest photon energy can be as high as $\sim 37 \mathrm{GeV}$, so it is plausible that $33 \mathrm{GeV}$ photon may result from synchrotron radiation.

(4) The wind parameter for three longest bursts is $A_{35.5} \sim 10^{-2}-10^{-1}$, which is reliably low. According to Dai \& Wu (2003), the progenitor star of GRB 080916C, GRB 090902B, and GRB 090926A could have been in a cloud. Due to a high pressure of the cloud, a speedy wind will be slowed down by a pair of shocks (viz, a reverse shock that propagates into the wind gas and a forward shock that propagates into the cloud). This interaction produces a stellar wind bubble and forms a density-jump at a radius of $\sim 10^{18} \mathrm{~cm}$. The homogeneous density outside this jump is estimated to be in the range of $n \sim 10^{-3} \mathrm{~cm}^{-3}$, which is much less than the typical density of an interstellar medium, but could not be excluded from the possibility of existence in areas of active star formation as the interiors of a prexisting superbubble (Panaitescu \& Kumar 2001; Scalo \& Wheeler 2001). If confirmed by the superbubble observation, this can provide a piece of evidence for a connection between long-duration GRBs and broad-lined SNe Ib/c. and would be natural to expect that massive progenitors of GRBs explode where we observe star formation. Meanwhile, in other fitting works (Cenko et al. 2010; Liu \& Wang 2010), they have also confronted with the problem of low density, and suggest the lower metallicity progenitors with minimal pre-explosion mass loss or selection effects (Cenko et al. 2010). Hence, although the LAT emission at least during $T_{90}$, from the spectral perspective (Zhang et al. 2010), is likely to connect to the GBM emission of internal origin, the fitting facts tentatively suggest a possibility that the LAT observation records an internal-external shock transition and at least in the LAT decaying phase the external forward shock emission is dominated.

\section{Conclusions and discussions}

It is widely believed that long duration gamma-ray bursts (GRBs), like hydrogendeficient Type Ib/c supernovae (SNe Ib/c), result from the core collapse of a massive star. The main characteristic of setting GRBs apart from other SNe is that a substantial fraction of the explosive energy is coupled to relativistic ejecta. A compact central engine is responsible for accelerating and collimating a jet-like outflow and driving a SN explosion 
(Woosley \& Bloom 2006; Gehrels et al. 2009; Soderberg et al. 2010). The precise nature of the central engine which powers GRB-SNe, however, remains an open question.

In this paper, we have undertaken extensive broadband $(>100 \mathrm{MeV}$, radio, optical, and X-ray) observations of three long-duration GRBs (GRB 080916C, GRB 090902B, GRB 090926A) detected by the LAT instrument on the Fermi satellite. The bulk Lorentz factors imply that the fireball is ultra-relativistic, which indicates that hyper-energetic bursts carry as high as $E_{i s o} \sim 10^{54}$ ergs in the blast wave. The temporal indices $\alpha$ and spectral indices $\beta$ in Tab. 1, which are consistent with the observed simple power law slopes, show that our detailed density transition consideration can reconcile the plateau and shallower decay in the later afterglow. Tab. 2 displays the estimated physical parameter space of the best fit, which is all reasonably certificated by the theory mentioned in Sec4. Specifically, the wind parameter $A_{35.5} \sim 10^{-2}-10^{-1}$ shed us light on the central engine of stellar collapsars and their association with SNe Ib/c (MacFadyen \& Woosley 1999; Woosley \& Heger 2006; Modjaz et al. 2008).

Furthermore, the tricky yet popular early rise of the LAT $(>100 \mathrm{MeV})$ light curve may indicate whether high energy photons share the same source as the low energy afterglows. Aside from two main kinds of explanation we mentioned above, leptonic or baryonic models, an alternative possibility comes from the high-latitude prompt emission (Kumar \& Panaitescu 2000) and thus the flux density evolves as $F_{\nu}(t) \propto t^{-2-\beta}$. However, according to $\beta_{o b, L A T}$ in Tab. 1, the calculated temporal index is too steep to explain the early high energy emission decay. Or else if the GeV component results from the same conventional unstructured ejecta as the late X-ray and optical afterglows, before the deceleration time ( $t<t_{d e c}$, that is, we only consider the thin shell case for long bursts), in the early wind bubble $n \propto R^{-2}$, the bulk Lorentz factor is nearly constant, and thus the electronic cooling and minimum frequencies evolve as $\nu_{c} \propto t$ and $\nu_{\min } \propto t^{-1}$, the peak flux density $F_{\max } \propto t^{0}$. Even when the SSC makes a significant effect, the IC electronic cooling and minimum frequencies and IC peak flux density evolve as $\nu_{I C, c} \propto t^{3}$ and $\nu_{I C, m} \propto t^{-1}$ and $F_{I C, \max } \propto t^{-1}$. Since $\max \left(\nu_{I C, c}, \nu_{I C, m}\right)>\nu_{G e V}>\max \left(\nu_{c}, \nu_{m}\right)$ around the peak of LAT light curve, $F_{\text {syn }, \mathrm{GeV}} \propto t^{(2-p) / 2}$ (for $3>p>2$, this slope is not enough), as well as $F_{I C, \mathrm{GeV}} \propto t^{-2 / 3}$ or $t^{-(p+1) / 3}$ for slow cooling and $F_{I C, G e V} \propto t^{-2}$ or $t^{-5 / 2}$ for fast cooling. No matter whether take the KN cutoff into account, this might be inconsistent with the observed rise of the LAT light curve.

Our explanation for the initial rising part is simply based on the assumption that $\Gamma \propto$ $t^{\varkappa}$. Therefore, $\nu_{c} \propto t^{\varkappa+1}, \nu_{m} \propto t^{-\varkappa-1}$, and $F_{\text {syn,GeV }} \propto e^{-\tau_{\gamma \gamma}} t^{(1+\varkappa)(2-p) / 2+2 \varkappa}$ when $\nu_{G e V}>$ $\max \left(\nu_{m}, \nu_{c}\right)$. Co-contribution of the optical depth and structured ejecta bring about the rising. This assumption can be understandable within the framework of of the core collapse 
of a massive star (i.e., SNe Ib/c). If the central engine ejects matter continuously, a clean tunnel would be left after the early ejecta sweeps up the stellar envelope, and the succeeding ejecta, whose energy is attained via continuous accretion even after the LAT trigger and whose Lorentz factor is larger, catches up with the early ejecta and injects more energy into the blast wave, giving rise to a rise of the LAT light curve. On the other hand, the thermal component can also be explained under such assumption that the previous jet lost kinetic energy due to impedance from shock frontier, then turned it into thermal radiation. This hypothesis have been provided by (MacFadyen et al. 2001) that, in the case of red-giant or blue super giants, the powerful jet lose its energy input at its base in the course of overtaking the sub-relativistic weak supernova shock before reaching the surface, and eject little highly relativistic matter; While in a helium star, the jet breaking out with continuously accretion power receiving at the base will lead its motion become highly relativistic. Later, some other possibilities, such as Zhang et al. (2003)'s baryon pollution scenario and Fan (2009)'s initial envelope choked model, also result in the earlier power-law rising distribution of bulk Lorentz factor.

In our frame, sub-MeV photons provide the background annihilation with the later $\mathrm{GeV}$ ones. On one hand, photon with the largest energy from the external shock fasten the constraint of lower Lorentz factor of the ejecta [in Granot (2010), the lower limit of the bulk Lorentz factor should be > 1000]; on the other hand, even the ejecta is slower than the lower limit, it is possible that the earlier rising is paretically contributed from opacity effect.

About the observed time delay of the pulses and variabilities in both LAT and GBM bands, some other modulation effects, except for optical depth can account for the concurrent variabilities in both bands, such as inhomogeneous surroundings, energy injection, etc.. These factors indicate that the variability connection between LAT and GBM (Zhang et al. 2010) cannot give a sufficient clue to the internal/external origin of the GeV photons.

In particular, as for GRB090902B, one of the tricky properties is its soft-hard-soft quasithermal spectrum evolution, since the soft-hard evolution appears within $T_{90}$, it can be better understood by our model that the initial outflow was dissipated as thermal component by the denser shock front/photosphere, when it breaks out of the envelope, the consequent highly relativistic ejecta in a clean tunnel can be dissipated strong enough to produce energetic non-thermal emission, even the photosphere can be outshined to some extent, therefore, the spectrum evolves from soft to hard.

This work is supported by the National Natural Science Foundation of China (grants 10873009 and 11033002) and the National Basic Research Program of China (973 program) No. 2007CB815404. 


\section{REFERENCES}

Abdo, A. A., et al. 2009, Science, 323, 1688

Abdo, A. A., et al. 2009, ApJ, 706, L138

Asano, K., Guiriec, S.,\& Mészáros, P. 2009, ApJ, 705, L191

Atwood, W. B., et al. 2009, ApJ, 697, 1071

Band, D., et al. 1993, ApJ, 413, 281

Bissaldi, E., \& Connaughton, V. 2009, GRB Coordinates Network, 9866, 1

Cenko, S. B., et al. 2011, ApJ, 732, 29

Chakrabarti, S. K., et al. 2009, GRB Coordinates Network, 10009, 1

Chandra, P., \& Frail, D.A. 2009, GRB Coordinates Network, 9889, 1

Chevalier, R. A., \& Li, Z.-Y. 2000, ApJ, 536, 195

Dai, Z. G., \& Lu, T. 1998a, A\&A, 333, L87

Dai, Z. G., \& Lu, T. 1998b, MNRAS, 298, 87

Dai, Z. G., \& Lu, T. 2002, ApJ, 565, L87

Dai, Z. G., \& Wu, X. F. 2003, ApJ, 591, L21

Evans, P. A., et al. 2007, A\&A, 469, 379

Evans, P. A., et al. 2009, MNRAS, 397, 1177

Evans, P. A., et al. 2009, GRB Coordinates Network, 9871, 1

Fan, Y.-Z. 2009, MNRAS, 397,1539

Fenimore, E. E., Madras, C. D., \& Nayakshin, S. 1996, ApJ, 473, 998

Gao, W.-H., Mao, J., Xu, D., \& Fan, Y.-Z. 2009, ApJ, 706, L33

Gehrels, N., et al. 2004, ApJ, 611, 1005

Gehrels, N., Ramirez-Ruiz, E., \& Fox, D. B. 2009, ARA\&A, 47, 567

Golenetskii, S., et al. 2009, GRB Coordinates Network, 9049, 1 
Granot, J., for the Fermi LAT Collaboration, \& the GBM Collaboration 2010, arXiv:1003.2452

Greiner, J., et al. 2009, A\&A, 498, 89

Gupta, N., \& Zhang, B. 2007, MNRAS, 380,78

Jin, Z. P., Xu, D., Covino,S., D'Avanzo, P., Antonelli, A., Fan, Y. Z., \& Wei, D. M. 2009, MNRAS, 400, 1829

Kennea, J. A., \& Stratta, G. 2009, GRB Coordinates Network, 9868, 1

Kumar, P., \& Barniol Duran, R. 2009, MNRAS, 400, L75

Kumar, P., \& Barniol Duran, R. 2010, MNRAS, 409, 226

Kumar, P., \& Panaitescu, A. 2000, ApJ, 541, L51

Lazzati, D., Rossi, E., Covino, S., Ghisellini, G., \& Malesani, D. 2002, A\&A, 396, L5

Li, Z. 2010, eprint arXiv, 1004, 791, 6 pages

Lithwick, Y., \& Sari, R. 2001, ApJ, 555, 540

Liu, R.Y., \& Wang, X.Y. 2011, ApJ, 730, 1

MacFadyen, A. I., \& Woosley, S. E. 1999, ApJ, 524, 262

MacFadyen, A. I., Woosley, S. E., \& Heger, A. 2001, ApJ, 550, 410

Maxham, B.B, Zhang, \& B.Zhang, 2010, (arXiv:1101.0144)

Mészáros, P., \& Rees, M. J. 1997, ApJ, 476, 232

Meegan, C., et al. 2009, ApJ, 702, 791

Modjaz, M., et al. 2008, AJ, 135, 1136

Nakar, E., Ando, S.\& Sari, R. 2009, ApJ, 703,675

Noda, K., et al. 2009, GRB Coordinates Network, 9951, 1

Panaitescu, A., \& Kumar, P. 2001, ApJ, 554,667

Panaitescu, A., \& Kumar, P. 2002, ApJ, 571, 779

Pandey, S. B., et al. 2010, ApJ, 714, 799 
Perri, M., Preger, B., \& Stratta, G. 2008, GRB Coordinates Network, 8261, 1

Piran, T., \& Nakar, E. 2010, ApJ, 718, L63

Rau, A., et al. 2010,ApJ, accepted (arXiv:1004.3261)

Razzaque, S., Dermer, C. D., \& Finke, J. D. 2009, arXiv:0908.0513

Rees, M. J., \& Meszaros, P. 1998,ApJ, 496, L1

Ryde, F., et al. 2010, ApJ, 709, L172

Sari, R. 1997, ApJ, 489, L37

Sari, R., Piran, T., \& Narayan, R. 1998, ApJ, 497, L17

Sari, R., \& Esin, A. A. 2001, ApJ, 548, 787

Scalo, J., \& Wheeler, J. C., 2001, ApJ, 562, 664

Soderberg, A. M., et al. 2010, Nature, 463, 513

Spergel, D. N., et al. 2007, ApJS, 170, 377

Stratta, G., D’Elia, V., \& Perri, M. 2009, GRB Coordinates Network, 9876, 1

Swenson, C. A., et al.\& Siegel, M. H. 2009, GRB Coordinates Network, 9877, 1

Swenson, C. A., et al. 2010, ApJ, 718, L14

Tam, P. H., Pun, C. S. J., Huang, Y. F., \& Cheng, K. S. 2005, New A, 10, 535

Terada, Y.,et al. 2009, GRB Coordinates Network, 9897, 1

Toma, K., Wu, X.-F., \& Mészáros, P. 2009, ApJ, 707, 1404

Uehara, T.,Takahashi,H., \& McEnery, J. 2009, GRB Coordinates Network, 9934, 1

van der Horst, A. J., Kamble, A. P., Wijers, R. A. M. J., \& Kouveliotou, C. 2009, GRB Coordinates Network, 9883, 1

Vetere, L. 2009, GRB Coordinates Network, 9961, 1

Wang, X. Y., Li,Z., Dai, Z. G.,\& Meszaros, P. 2009, ApJ, 698, 198

Wang, X. Y., He,H N., Li,Z.,\& Dai, Z. G. 2010, ApJ, 712, 1232 
Woosley, S. E., \& Bloom, J. S. 2006, ARA\&A, 44, 507

Woosley, S. E., \& Heger, A. 2006, ApJ, 637, 9145

Wu, X. F., Dai, Z. G.,Huang, Y. F., \& Lu, T. 2005, ApJ, 619, 968

Zhang, W., Woosley, S. E., \& Heger, A. 2008, ApJ, 679, 639

Zhang, B., \& Pe'er, A. 2009, ApJ, 700,L65

Zhang, B.-B., et al. 2010, ApJ, 730, 141

Zou, Y.-C., Fan, Y.-Z.,\& Piran, T. 2009, MNRAS, 396, 1163

Zou, Y.-C., Fan, Y.-Z.,\& Piran, T. 2011, ApJ, 726, L2 


\begin{tabular}{|c|c|c|c|c|c|c|c|c|c|c|c|}
\hline GRBs & $\alpha_{L A T}$ & $\alpha_{o b, L A T}$ & $\beta_{L A T}$ & $\beta_{o b, L A T}$ & $\alpha_{x, o, 1}$ & $\alpha_{x, o, 2}$ & $\alpha_{o b, x}$ & $\alpha_{o b, o}$ & $\beta_{x, o}$ & $\beta_{o b, x}$ & $\beta_{o b, o}$ \\
\hline $080916 C^{a}$ & 1.27 & $1.33 \pm 0.08$ & 1.16 & $1.1 \pm 0.1$ & 1.49 & 0.99 & $1.29 \pm 0.09$ & $1.40 \pm 0.05$ & 0.66 & $0.50 \pm 0.16$ & $0.38 \pm 0.02$ \\
\hline $090902 B^{b}$ & 1.33 & $1.40 \pm 0.06$ & 1.2 & $-*$ & 1.49 & 0.98 & $1.36 \pm 0.03$ & $0.89 \pm 0.05$ & 0.7 & $0.90 \pm 0.13$ & $0.76 \pm 0.07$ \\
\hline $090926 A^{c}$ & 2.01 & $2.05 \pm 0.14$ & 1.5 & $1.26_{-0.24}^{+0.22}$ & 2.00 & 1.50 & $1.43 \pm 0.03^{\star}$ & $1.38 \pm 0.02^{\star}$ & 1.00 & $1.12 \pm 0.13^{\star}$ & $1.03 \pm 0.05^{\star}$ \\
\hline
\end{tabular}

Table 1: According to $F_{\nu} \propto t^{-\alpha} \nu^{-\beta}, \alpha_{L A T}, \alpha_{x, o, 1}, \alpha_{x, o, 2}$ are the estimated temporal indices of the LAT band photons, $\mathrm{X}$-ray + optical photons in the wind bubble, and X-ray + optical photons in the homogeneous medium from our best-fit parameters. $\alpha_{o b, L A T}, \alpha_{o b, x}, \alpha_{o b, o}$ are the observed temporal indices of $\mathrm{GeV}$ photons, simple power-law X-ray photons and optical photons. $\beta_{L A T}$ and $\beta_{x, o}$ are the estimated spectral indices of the LAT band photons and X-ray + optical photons in either medium from our best-fit parameters. $\beta_{o b, L A T}, \beta_{o b, x}$, and $\beta_{o b, o}$ are the observed spectral indices of GeV photons, simple power-law X-ray photons and optical photons. High energy data are taken from Zhang et al. (2010), X-ray data are taken from Swift Group (Evans et al. 2007, 2009). In addition, the data labeled with superscript $a$ are taken from Greiner et al. (2009), the data with $b$ are from Pandey et al. (2010), the data with $c$ are from Swenson et al. (2010),

represents no data available. For 090926A, the data labeled with $\star$ are from Cenko et al. (2010), the temporal slope of the X-ray and optical band in Rau et al. (2010) is: $\alpha=1.6 \pm 0.2$ before the first flare, $\alpha=1.75 \pm 0.04$ after the second flare, and $\alpha=1.63 \pm 0.01$ between, which is consistent with our estimated value.

\begin{tabular}{c|cccccccccccccc}
\hline \hline GRBs & $p$ & $\varkappa$ & $E_{K, \text { iso }, 55}$ & $\eta_{3}$ & $A_{\star, 35.5}$ & $\epsilon_{e}$ & $\epsilon_{B}$ & $\xi$ & $t_{\text {break }}(\mathrm{s})$ & $t_{\text {end }}(\mathrm{s})$ & $t_{\text {jet }}(\mathrm{s})$ & $\varsigma_{\gamma}$ & $R_{\text {break }}(\mathrm{cm})$ & $\left.n_{a f t e r}(\mathrm{~cm})^{-3}\right)$ \\
\hline $080916 C$ & 2.32 & 0.5 & 0.13 & 0.74 & 0.02 & 0.3 & $5.81 \times 10^{-6}$ & 2.1 & $10^{5.3}$ & $10^{5.6}$ & - & $87.1 \%$ & $3.95 \times 10^{18}$ & $1.17 \times 10^{-3}$ \\
$090902 B$ & 2.4 & 0.8 & 0.44 & 0.60 & 0.06 & 0.4 & $4.56 \times 10^{-7}$ & 2.0 & $10^{5.1}$ & $10^{6.0}$ & $10^{6.0}$ & $46.4 \%$ & $7.52 \times 10^{18}$ & $1.62 \times 10^{-3}$ \\
$090926 \mathrm{~A}$ & 3.0 & 0.3 & 0.43 & 0.56 & 0.03 & 0.35 & $8.18 \times 10^{-6}$ & 2.5 & $10^{4.8}$ & $10^{4.9}$ & - & $33.0 \%$ & $4.67 \times 10^{18}$ & $1.31 \times 10^{-3}$ \\
\hline \hline
\end{tabular}

Table 2: The left part of the table are best-fit parameters for GRB080916C, GRB090902B, and GRB090926A, the right part are prompt emission energy fraction, radius when a density jump occurs and circumburst density after the jump, respectively. 

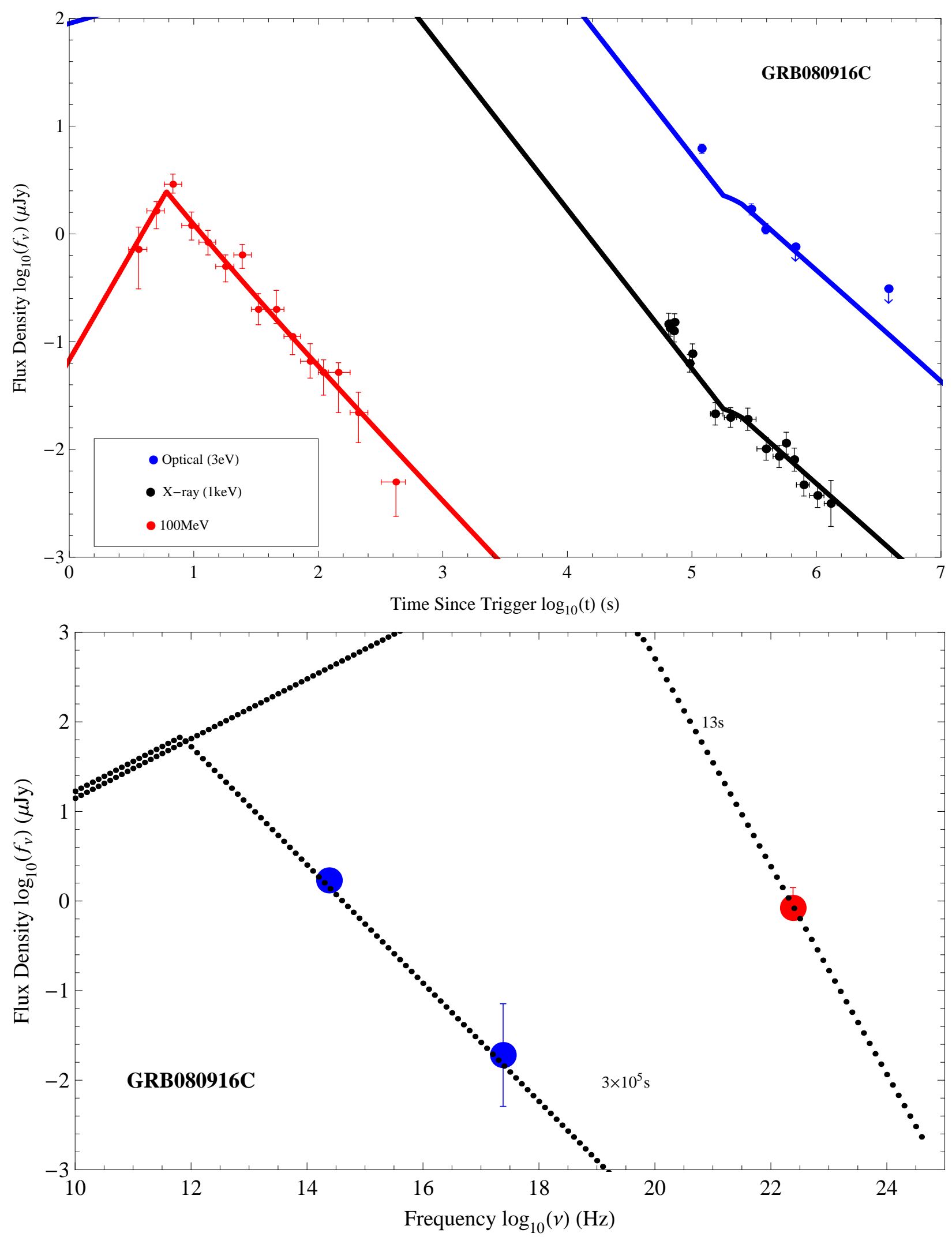

Fig. 1.- Best fit for GRB 080916C: $p=2.32, \varkappa=0.5, E_{K, 55}^{i s o}=0.13, \eta_{3}=0.74, A_{35.5}=0.02$, $\epsilon_{e, 0}=0.3, \epsilon_{B, 0}=5.81 \times 10^{-6}, \varepsilon=2.1, t_{\text {break }}=10^{5.3} \mathrm{~s}, t_{\text {end }}=10^{5.6} \mathrm{~s}$, no jet break appears during the modeling period 


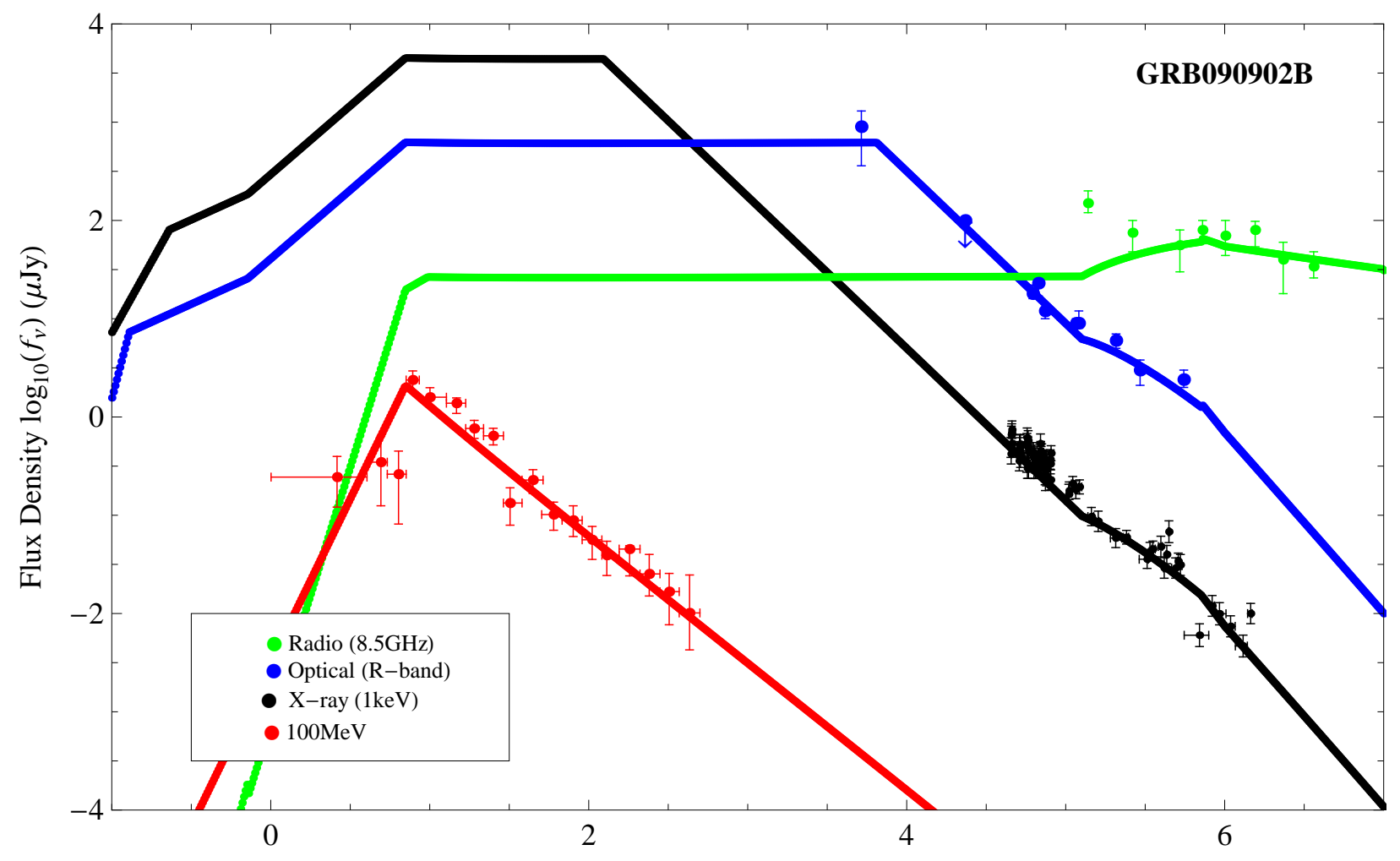

Time Since Trigger $\log _{10}(\mathrm{t})(\mathrm{s})$

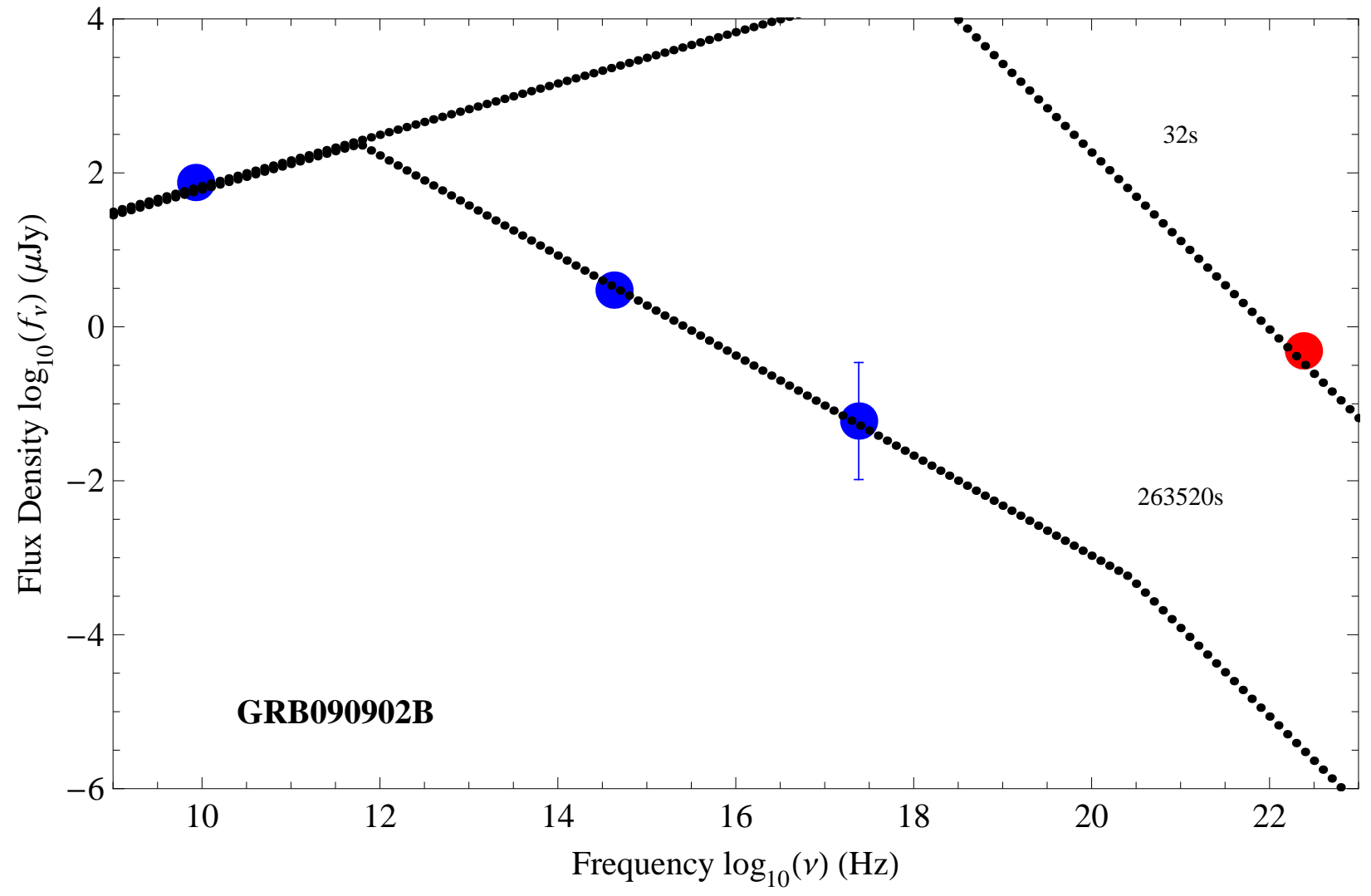

Fig. 2.- Best fit for GRB 090902B: $p=2.4, \varkappa=0.8, E_{K, 55}^{i s o}=0.44, \eta_{3}=0.60, A_{35.5}=0.06$, $\epsilon_{e, 0}=0.4, \epsilon_{B, 0}=4.56 \times 10^{-7}, \varepsilon=2.0, t_{\text {break }}=10^{5.1} \mathrm{~s}, t_{\text {end }}=10^{6.0} \mathrm{~s}, t_{\text {jet }}=10^{6.0} \mathrm{~s}$ 

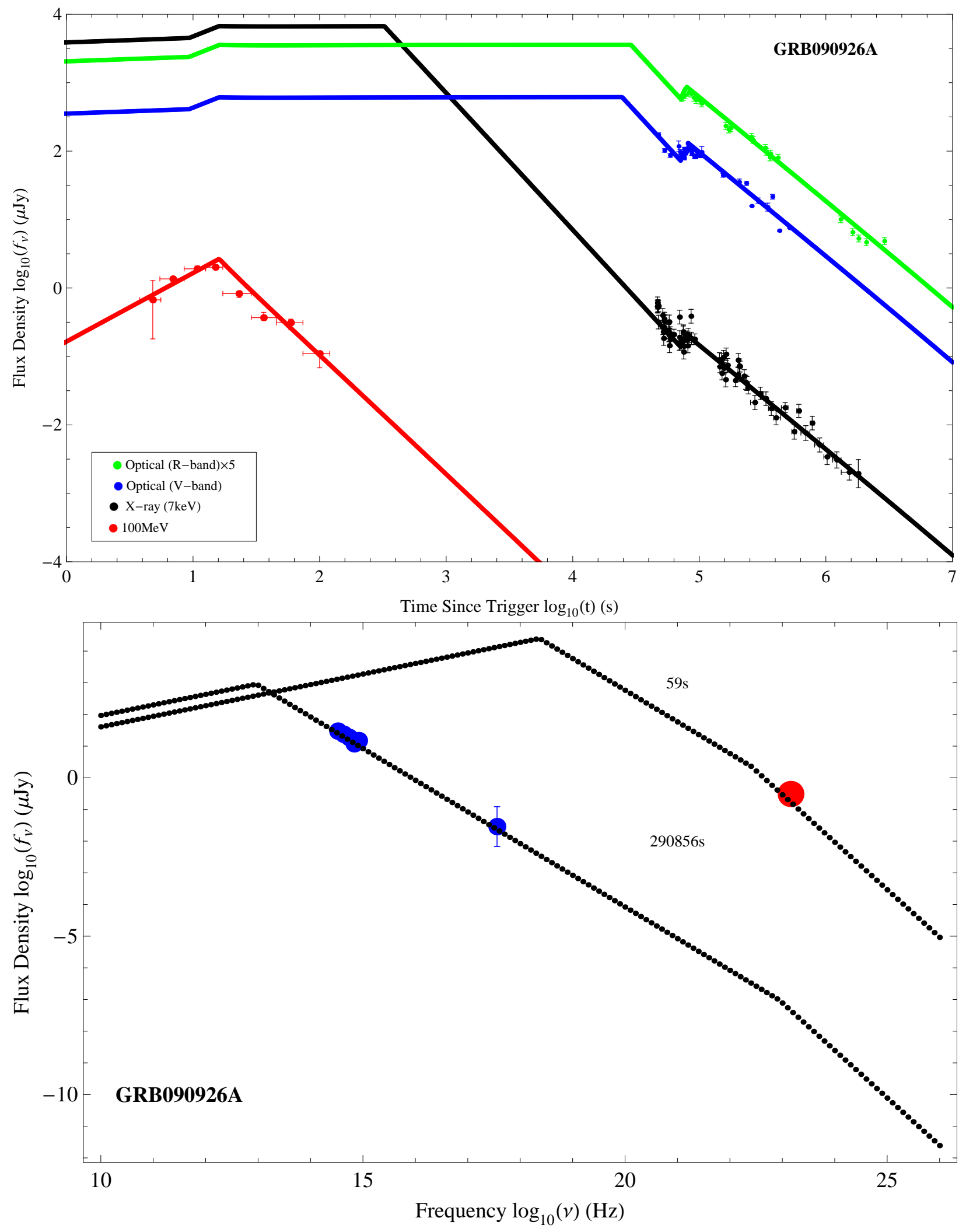

Fig. 3.- Best fit for GRB 090926A: $p=3.0, \varkappa=0.3, E_{K, 55}^{i s o}=0.43, \eta_{3}=0.56, A_{35.5}=0.03$, $\epsilon_{e, 0}=0.35, \epsilon_{B, 0}=8.18 \times 10^{-6}, \varepsilon=2.5, t_{\text {break }}=10^{4.8} \mathrm{~s}, t_{\text {end }}=10^{4.9} \mathrm{~s}$, no jet break confirmed until 21 days 

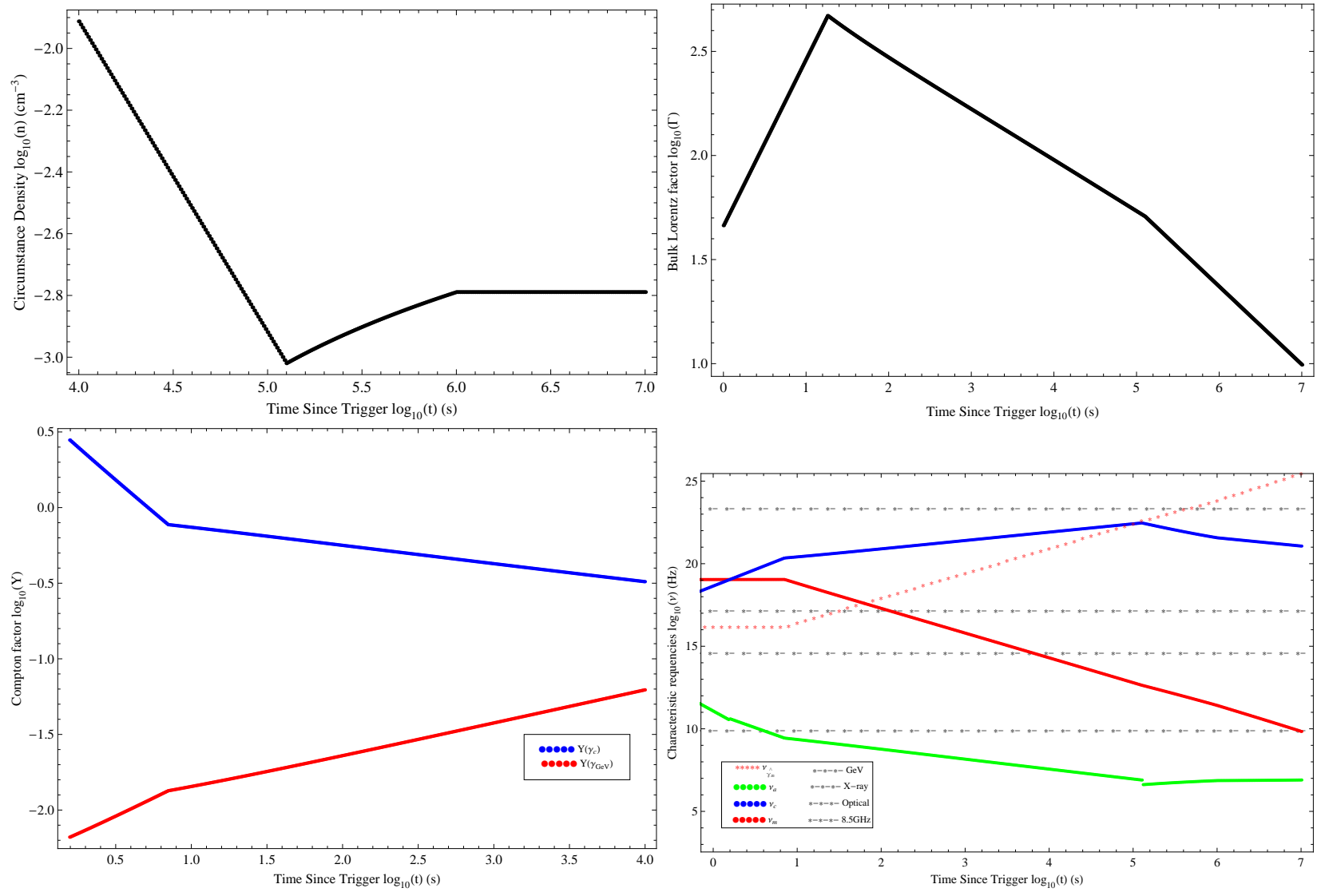

Fig. 4.- Physical parameter evolution with the best fit parameter for GRB090902B, similar to the other two bursts. Upper-left panel: a. circumburst density with radius, upper-right panel: b. blast wave bulk Lorentz factor with time; lower-left panel: c. Compton $Y$ factor ( $\ll 1$ due to KN effects) with time; lower-right panel: d. characteristic frequency evolution. 